\title{
A New Solvent-Dependent Mechanism for a Triazolinedione Ene Reaction
}

\section{Orlando Acevedo and Michael Squillacote}

Contribution from the Department of Chemistry and Biochemistry, Auburn University, Auburn, Alabama 36849.

Supporting Material

Figure S1. Free-energy map 1 for the ene reaction in methanol and acetonitrile.................2

Figure S2. Free-energy map 2 for the ene reaction in methanol and acetonitrile.................2

Figure S3. Ene Reaction between PTAD and Tetramethylethylene in DMSO.....................3

Figure S4. Solute-Solvent Energy Pair Distribution in DMSO and Methanol......................4

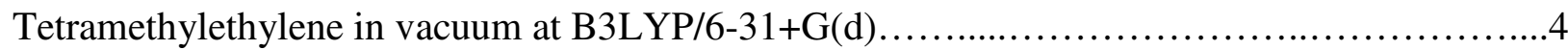

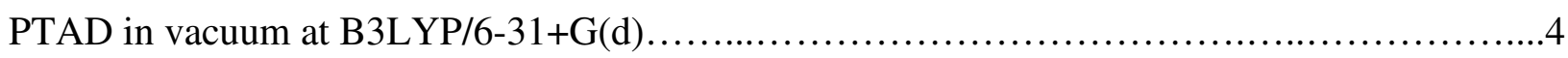

Transition structure, $\mathrm{TS}_{\text {add. }}$, in vacuum at B3LYP/6-31+G(d) ..................................

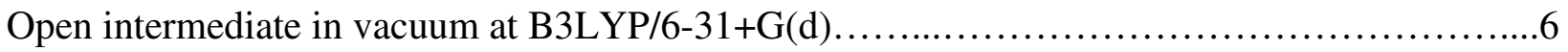

Intermediate isomerization transition structure in vacuum at $\mathrm{B} 3 \mathrm{LYP} / 6-31+\mathrm{G}(\mathrm{d}) \ldots \ldots \ldots \ldots \ldots \ldots . .7$

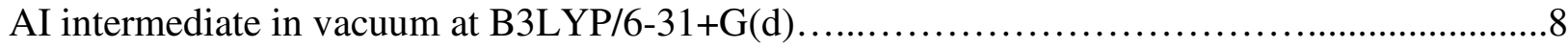

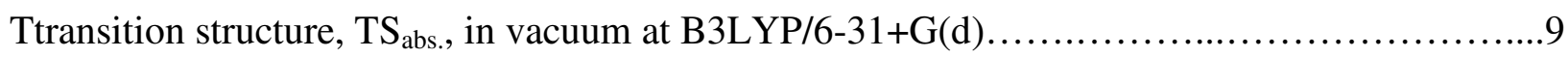

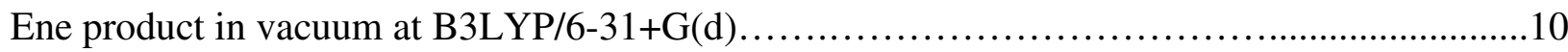

Tetramethylethylene in solution at B3LYP/6-311++G(2d,p) with CPCM....................10-11

PTAD in solution at B3LYP/6-311++G(2d,p) with CPCM ..............................12-13

Transition structure, $\mathrm{TS}_{\text {add. }}$, in solution at B3LYP/6-311++G(2d,p) with CPCM .............13-14

Open intermediate in solution at B3LYP/6-311++G(2d,p) with CPCM ....................15-16

Transition structure, $\mathrm{TS}_{\text {isomer. }}$, in solution at B3LYP/6-311++G(2d,p) with CPCM .......... 16-17

AI intermediate in solution at B3LYP/6-311++G(2d,p) with CPCM ........................18-19 
Transition structure, $\mathrm{TS}_{\mathrm{abs}}$, in solution at B3LYP/6-311++G(2d,p) with CPCM $19-20$

Ene product in solution at B3LYP/6-311++G(2d,p) with CPCM 20-21

Open intermediate in vacuum at UB3LYP/6-31+G(d) .22

Gaussian 03 reference. 23

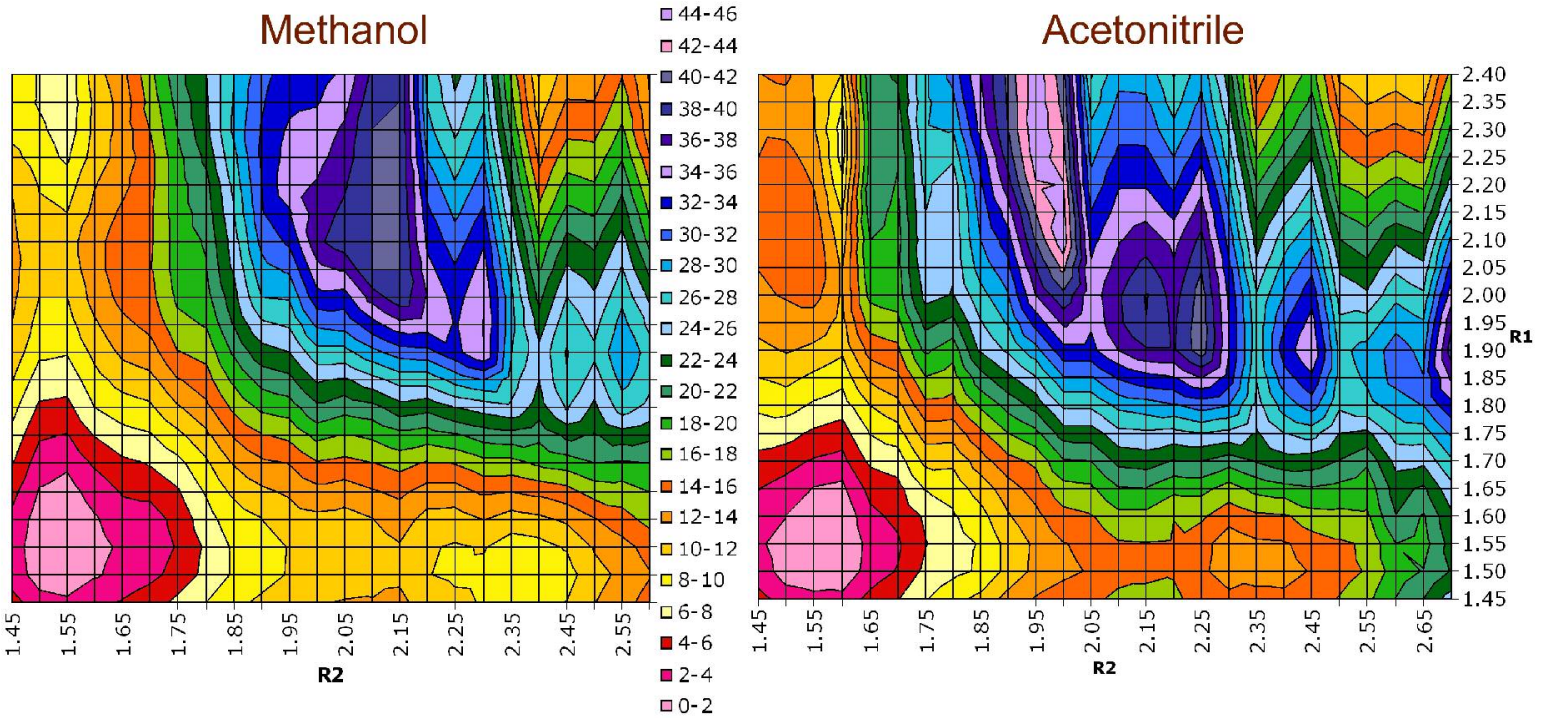

Figure S1. Two-dimensional potentials of mean force (free-energy map 1; reaction coordinates $\mathrm{R}_{1}$ and $\mathrm{R}_{2}$ ) for the ene reaction in methanol and acetonitrile. All distances in angstroms and relative free-energies in $\mathrm{kcal} / \mathrm{mol}$.
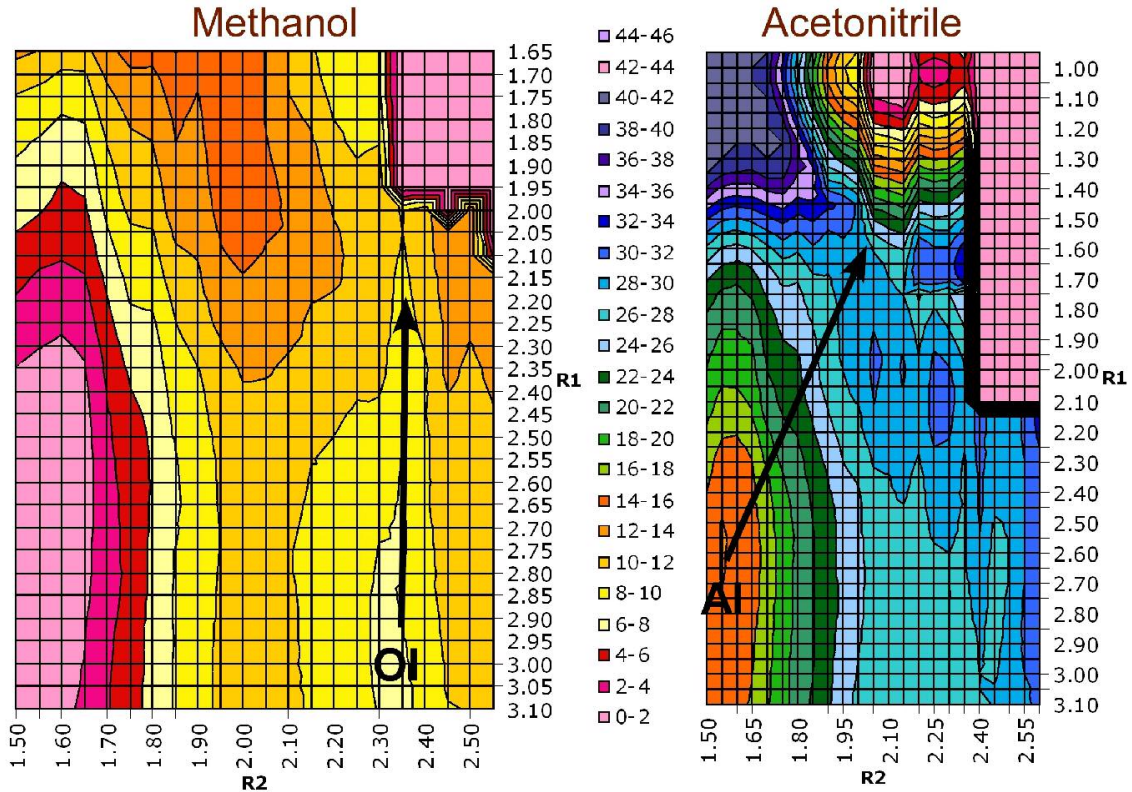

Figure S2. Two-dimensional potentials of mean force (free-energy map 2; reaction coordinates $\mathrm{R}_{2}$ and $\mathrm{R}_{\mathrm{NH}}$ ) for the ene reaction in methanol and acetonitrile. All distances in angstroms and relative freeenergies in $\mathrm{kcal} / \mathrm{mol}$. $\mathrm{AI}=$ aziridinium imide intermediate, $\mathrm{OI}=$ open intermediate. 


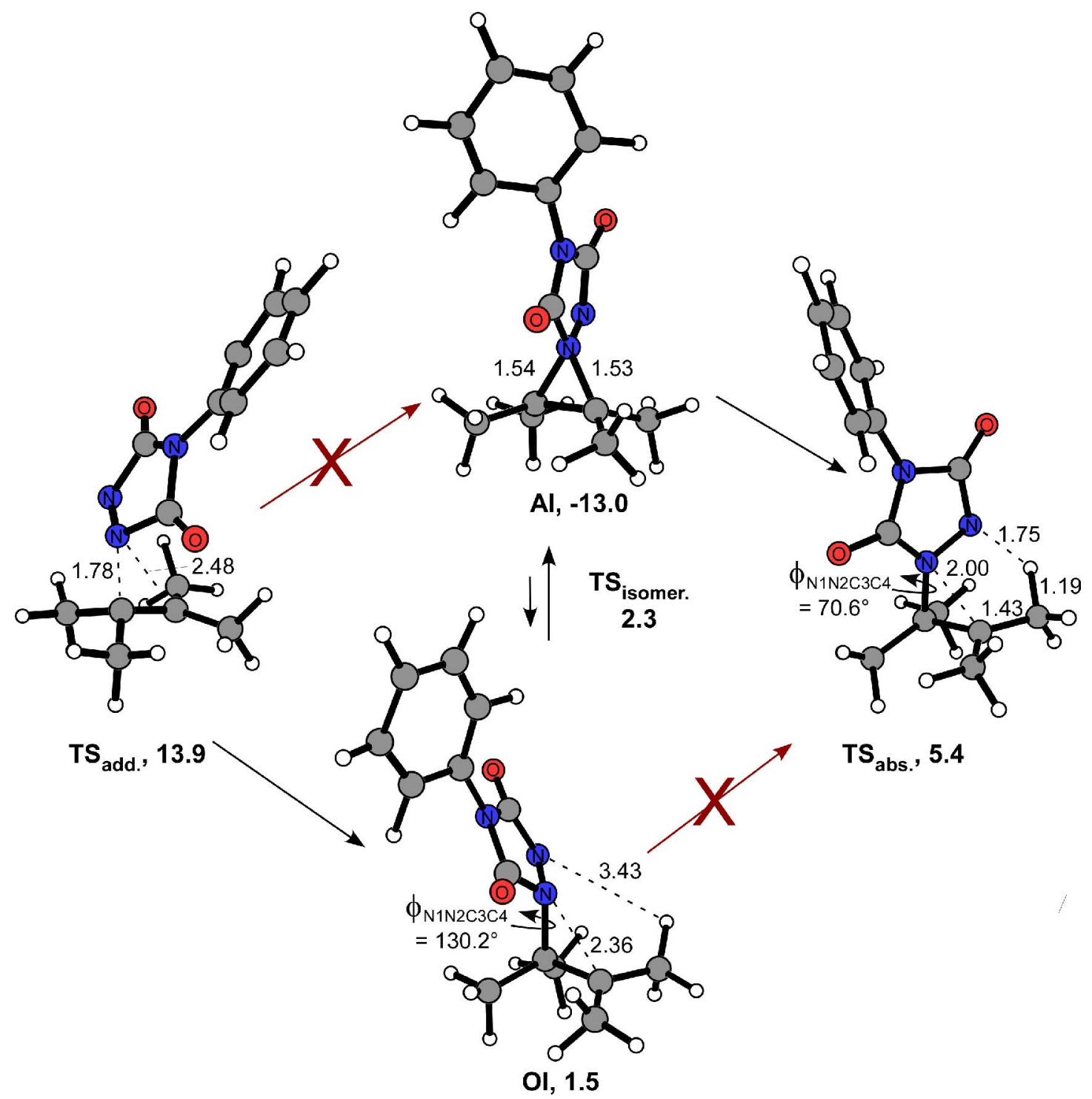

Figure S3. Optimized structures for the ene reaction between 4-phenyl-1,2,4-triazoline-3,5-dione (PTAD) and tetramethylethylene in DMSO from $\mathrm{QM} / \mathrm{MM} / \mathrm{MC}$ simulations. $\mathrm{AI}=$ aziridinium imide, $\mathrm{OI}=$ open intermediate, $\mathrm{TS}_{\mathrm{add} .}=$ addition of PTAD, $\mathrm{TS}_{\mathrm{abs} .}=$ allylic hydrogen abstraction, $\mathrm{TS}_{\text {isomer. }}=$ isomerization between intermediates, $\mathrm{X}=$ energetically-unfavorable reaction path. All distances in angstroms, and free energies relative to the reactants (shown in bold) in $\mathrm{kcal} / \mathrm{mol}$. 


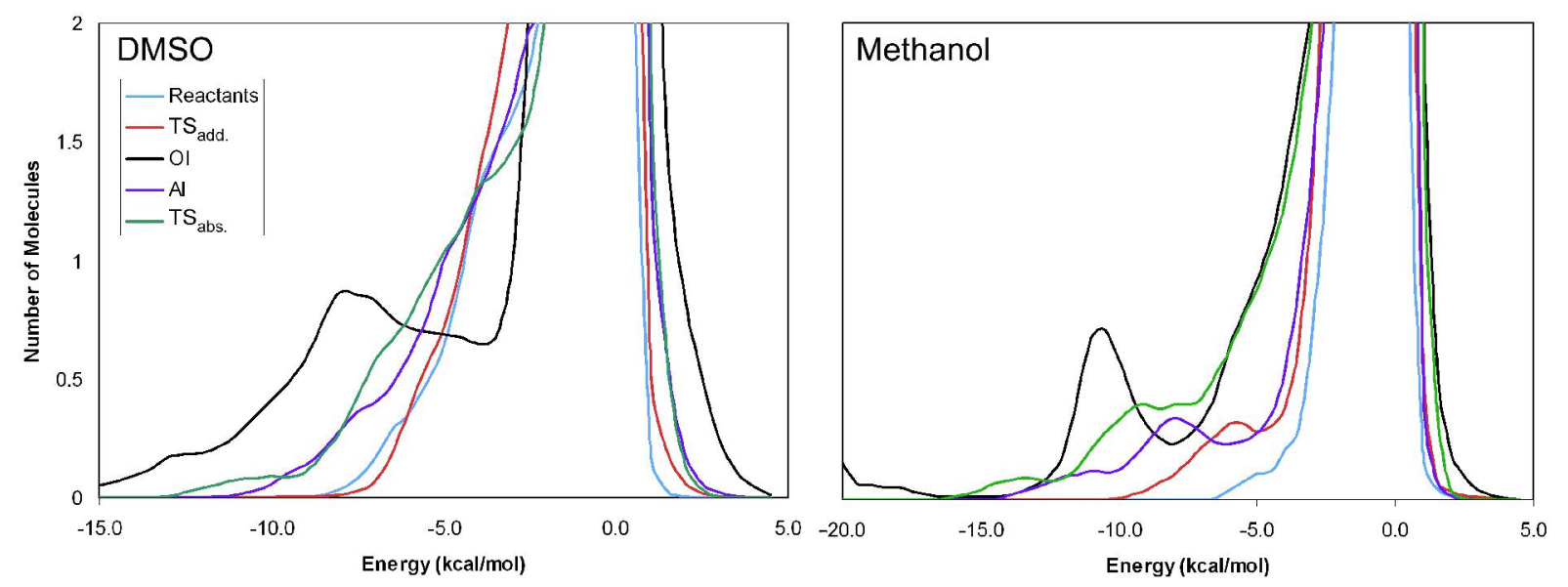

Figure S4. Solute-solvent energy pair distributions for the reaction between PTAD and tetramethylethylene in DMSO and methanol for the reactants, transition structures $\left(\mathrm{TS}_{\mathrm{add} .}\right.$ and $\mathrm{TS}_{\mathrm{abs}}$.), aziridinium imide (AI), and open intermediate (OI). The ordinate records the number of solvent molecules that interact with the solutes with their interaction energy on the abscissa. Units for the ordinate are number of molecules per $\mathrm{kcal} / \mathrm{mol}$.

\section{Tetramethylethylene from B3LYP/6-31+G(d)}

$1 \backslash 1 \backslash$ ASN_ALTIX - ALTIX2 $\backslash$ Freq $\backslash R B 3 L Y P \backslash 6-31+G(d) \backslash C 6 H 12 \backslash A U B O X A \backslash 11-A p r-2007 \backslash 1$ $\backslash \backslash \#$ B3LYP/6-31+G(d) freq guess=read \\Tetramethylethylene $\backslash \backslash 0,1 \backslash C \backslash C, 1, B 1$ $\backslash \mathrm{C}, 2, \mathrm{~B} 2,1, \mathrm{~A} 1 \backslash \mathrm{H}, 3, \mathrm{~B} 3,2, \mathrm{~A} 2,1, \mathrm{D} 1,0 \backslash \mathrm{H}, 3, \mathrm{~B} 4,2, \mathrm{~A} 3,1, \mathrm{D} 2,0 \backslash \mathrm{H}, 3, \mathrm{~B} 5,2, \mathrm{~A} 4,1, \mathrm{D} 3,0 \backslash$ $\mathrm{C}, 2, \mathrm{~B} 6,1, \mathrm{~A} 5,3, \mathrm{D} 4,0 \backslash \mathrm{H}, 7, \mathrm{~B} 7,2, \mathrm{~A} 6,1, \mathrm{D} 5,0 \backslash \mathrm{H}, 7, \mathrm{~B} 8,2, \mathrm{~A} 7,1, \mathrm{D} 6,0 \backslash \mathrm{H}, 7, \mathrm{~B} 9,2, \mathrm{~A} 8,1$

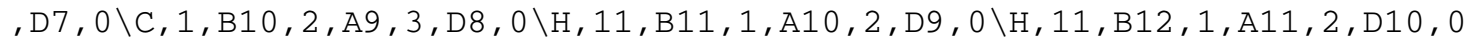
$\backslash \mathrm{H}, 11, \mathrm{~B} 13,1, \mathrm{~A} 12,2, \mathrm{D} 11,0 \backslash \mathrm{C}, 1, \mathrm{~B} 14,11, \mathrm{~A} 13,2, \mathrm{D} 12,0 \backslash \mathrm{H}, 15, \mathrm{~B} 15,1, \mathrm{~A} 14,11, \mathrm{D} 13,0$ $\backslash \mathrm{H}, 15, \mathrm{~B} 16,1, \mathrm{~A} 15,11, \mathrm{D} 14,0 \backslash \mathrm{H}, 15, \mathrm{~B} 17,1, \mathrm{~A} 16,11, \mathrm{D} 15,0 \backslash \backslash \mathrm{B} 1=1.3519 \backslash \mathrm{B} 2=1.5153 \backslash$ $B 3=1.091 \backslash B 4=1.0998 \backslash B 5=1.0998 \backslash B 6=1.5153 \backslash B 7=1.0998 \backslash B 8=1.0998 \backslash B 9=1.091 \backslash B 1$ $0=1.5153 \backslash B 11=1.091 \backslash B 12=1.0998 \backslash B 13=1.0998 \backslash B 14=1.5153 \backslash B 15=1.091 \backslash B 16=1.09$ $98 \backslash B 17=1.0999 \backslash A 1=124.4068 \backslash A 2=114.4373 \backslash A 3=110.3675 \backslash A 4=110.3673 \backslash A 5=124.3$ $966 \backslash A 6=110.366 \backslash A 7=110.3668 \backslash A 8=114.4366 \backslash A 9=124.3918 \backslash A 10=114.4308 \backslash A 11=11$ $0.3723 \backslash \mathrm{A} 12=110.3668 \backslash \mathrm{A} 13=111.2017 \backslash \mathrm{A} 14=114.4345 \backslash \mathrm{A} 15=110.3712 \backslash \mathrm{A} 16=110.366$ $4 \backslash D 1=0.0134 \backslash D 2=121.4644 \backslash D 3=-121.4329 \backslash D 4=-180.0054 \backslash D 5=121.4477 \backslash D 6=-121$. $4496 \backslash D 7=0.001 \backslash D 8=180.0012 \backslash D 9=0.0021 \backslash D 10=121.4499 \backslash D 11=-121.4433 \backslash D 12=179$ $.9996 \backslash \mathrm{D} 13=-180.0121 \backslash \mathrm{D} 14=-58.5597 \backslash \mathrm{D} 15=58.5454 \backslash \backslash$ Version=IA64L $-\mathrm{G} 03$ RevD .01 $\backslash$ State $=1-A \backslash H F=-235.8639896 \backslash R M S D=4.342 e-09 \backslash R M S F=1.722 e-05 \backslash$ zeroPoint $=0.1$ $642108 \backslash$ Thermal $=0.1729288 \backslash$ Dipole $=0.0000223,-0.0000216,0.0000574$

Zero-point correction=

Thermal correction to Energy=

Thermal correction to Enthalpy=

Thermal correction to Gibbs Free Energy=

Sum of electronic and zero-point Energies=

Sum of electronic and thermal Energies=

Sum of electronic and thermal Enthalpies=

Sum of electronic and thermal Free Energies=

\author{
0.164211 (Hartree/Particle) \\ 0.172929 \\ 0.173873 \\ 0.130636 \\ $-235.699779$ \\ $-235.691061$ \\ $-235.690117$ \\ $-235.733353$
}

\section{PTAD from B3LYP/6-31+G(d)}

1 | 1 | UNPC - UNK | Freq | RB3LYP | 6 - 31+G (d) | C8H5N302 | PCUSER | 12-Apr-2007|1||\# B3 LYP / 6-31+G (D) FREQ GUESS=READ ||PhTAD ||0,1|N|N, 1, B1 |C, 2, B2, 1, A1 |C, 1, B3, 2, $\mathrm{A} 2,3, \mathrm{D} 1,0|\mathrm{~N}, 4, \mathrm{~B} 4,1, \mathrm{~A} 3,2, \mathrm{D} 2,0| \mathrm{O}, 3, \mathrm{~B} 5,2, \mathrm{~A} 4,1, \mathrm{D} 3,0|\mathrm{O}, 4, \mathrm{~B} 6,1, \mathrm{~A} 5,2, \mathrm{D} 4,0| \mathrm{C}$ , $5, \mathrm{~B} 7,4, \mathrm{~A} 6,1, \mathrm{D} 5,0|\mathrm{C}, 8, \mathrm{~B} 8,5, \mathrm{~A} 7,4, \mathrm{D} 6,0| \mathrm{C}, 8, \mathrm{~B} 9,5, \mathrm{~A} 8,4, \mathrm{D} 7,0 \mid \mathrm{C}, 9, \mathrm{~B} 10,8, \mathrm{~A} 9,5$ 
, D8 , 0 | C , 10 , B11, 8, A10 , 5 , D9 , $0|\mathrm{C}, 11, \mathrm{~B} 12,9, \mathrm{~A} 11,8, \mathrm{D} 10,0| \mathrm{H}, 10, \mathrm{~B} 13,8, \mathrm{~A} 12,5, \mathrm{D} 1$ $1,0|\mathrm{H}, 9, \mathrm{~B} 14,8, \mathrm{~A} 13,5, \mathrm{D} 12,0| \mathrm{H}, 12, \mathrm{~B} 15,10, \mathrm{~A} 14,8, \mathrm{D} 13,0 \mid \mathrm{H}, 11, \mathrm{~B} 16,9, \mathrm{~A} 15,8, \mathrm{D} 14$ , $0|\mathrm{H}, 13, \mathrm{~B} 17,11, \mathrm{~A} 16,9, \mathrm{D} 15,0||\mathrm{B} 1=1.2413| \mathrm{B} 2=1.5001|\mathrm{~B} 3=1.5| \mathrm{B} 4=1.3908 \mid \mathrm{B} 5=1$. $1991|\mathrm{~B} 6=1.1991| \mathrm{B} 7=1.4344|\mathrm{~B} 8=1.3977| \mathrm{B} 9=1.3977|\mathrm{~B} 10=1.3959| \mathrm{B} 11=1.3959 \mid \mathrm{B} 12$ $=1.3974|\mathrm{~B} 13=1.0846| \mathrm{B} 14=1.0846|\mathrm{~B} 15=1.0863| \mathrm{B} 16=1.0863|\mathrm{~B} 17=1.0865| \mathrm{A} 1=109$. $735|\mathrm{~A} 2=109.7396| \mathrm{A} 3=106.1138|\mathrm{~A} 4=123.8546| \mathrm{A} 5=123.8691|\mathrm{~A} 6=125.8352| \mathrm{A} 7=119$ $.4406|\mathrm{~A} 8=119.4631| \mathrm{A} 9=119.1395|\mathrm{~A} 10=119.1366| \mathrm{A} 11=120.372|\mathrm{~A} 12=120.3186| \mathrm{A} 1$ $3=120.3117|\mathrm{~A} 14=119.3797| \mathrm{A} 15=119.3812|\mathrm{~A} 16=120.0638| \mathrm{D} 1=0.127|\mathrm{D} 2=-0.0576|$ $\mathrm{D} 3=-180.3027|\mathrm{D} 4=179.7543| \mathrm{D} 5=180.234|\mathrm{D} 6=-45.6844| \mathrm{D} 7=134.3038 \mid \mathrm{D} 8=-180.31$ $38|\mathrm{D} 9=179.7088| \mathrm{D} 10=0.6054|\mathrm{D} 11=0.3177| \mathrm{D} 12=0.2798|\mathrm{D} 13=-179.6602| \mathrm{D} 14=-179$ $.669|\mathrm{D} 15=179.6937| \mid$ Version=x86-Win32-G03RevB $01 \mid$ State=1-A $\mid \mathrm{HF}=-622.5455$ $042|\mathrm{RMSD}=6.201 \mathrm{e}-009| \mathrm{RMSF}=3.720 \mathrm{e}-005 \mid \mathrm{Dipole}=1.5496764,-0.0000048,-0.000$ 7586

Zero-point correction=

Thermal correction to Energy=

Thermal correction to Enthalpy=

Thermal correction to Gibbs Free Energy=

Sum of electronic and zero-point Energies=

Sum of electronic and thermal Energies=

Sum of electronic and thermal Enthalpies=

Sum of electronic and thermal Free Energies=
0.124551 (Hartree/Particle)

0.134368

0.135313

0.088020

$-622.420953$

$-622.411136$

$-622.410192$

$-622.457484$

\section{Transition Structure, TS add., from B3LYP/6-31+G(d)}

$1 \backslash 1 \backslash$ ASN_ALTIX - ALTIX5 \Freq \RB3LYP \6-31+G (d) \C14H17N3O2\AUBOXA \05-Apr - 2

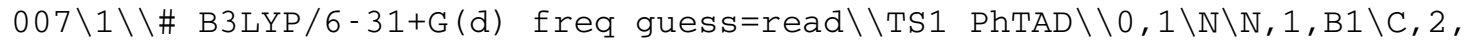
$\mathrm{B} 2,1, \mathrm{~A} 1 \backslash \mathrm{C}, 1, \mathrm{~B} 3,2, \mathrm{~A} 2,3, \mathrm{D} 1,0 \backslash \mathrm{N}, 3, \mathrm{~B} 4,2, \mathrm{~A} 3,1, \mathrm{D} 2,0 \backslash 0,3, \mathrm{~B} 5,2, \mathrm{~A} 4,1, \mathrm{D} 3,0 \backslash 0,4, \mathrm{~B}$ $6,1, \mathrm{~A} 5,2, \mathrm{D} 4,0 \backslash \mathrm{C}, 5, \mathrm{~B} 7,3, \mathrm{~A} 6,2, \mathrm{D} 5,0 \backslash \mathrm{C}, 8, \mathrm{~B} 8,5, \mathrm{~A} 7,3, \mathrm{D} 6,0 \backslash \mathrm{C}, 8, \mathrm{~B} 9,5, \mathrm{~A} 8,3, \mathrm{D} 7,0$ $\backslash \mathrm{C}, 9, \mathrm{~B} 10,8, \mathrm{~A} 9,5, \mathrm{D} 8,0 \backslash \mathrm{C}, 10, \mathrm{~B} 11,8, \mathrm{~A} 10,5, \mathrm{D} 9,0 \backslash \mathrm{C}, 12, \mathrm{~B} 12,10, \mathrm{~A} 11,8, \mathrm{D} 10,0 \backslash \mathrm{H}, 1$ $0, \mathrm{~B} 13,8, \mathrm{~A} 12,5, \mathrm{D} 11,0 \backslash \mathrm{H}, 9, \mathrm{~B} 14,8, \mathrm{~A} 13,5, \mathrm{D} 12, \mathrm{O} \backslash \mathrm{H}, 12, \mathrm{~B} 15,10, \mathrm{~A} 14,8, \mathrm{D} 13,0 \backslash \mathrm{H}, 11$

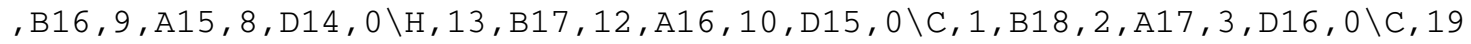

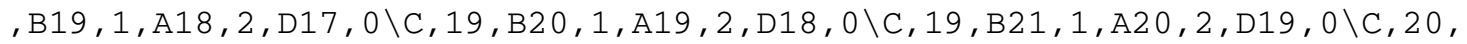
$\mathrm{B} 22,19, \mathrm{~A} 21,1, \mathrm{D} 20,0 \backslash \mathrm{C}, 20, \mathrm{~B} 23,19, \mathrm{~A} 22,1, \mathrm{D} 21,0 \backslash \mathrm{H}, 21, \mathrm{~B} 24,19, \mathrm{~A} 23,1, \mathrm{D} 22,0 \backslash \mathrm{H}, 2$ $1, \mathrm{~B} 25,19, \mathrm{~A} 24,1, \mathrm{D} 23,0 \backslash \mathrm{H}, 21, \mathrm{~B} 26,19, \mathrm{~A} 25,1, \mathrm{D} 24,0 \backslash \mathrm{H}, 22, \mathrm{~B} 27,19, \mathrm{~A} 26,1, \mathrm{D} 25,0 \backslash \mathrm{H}$

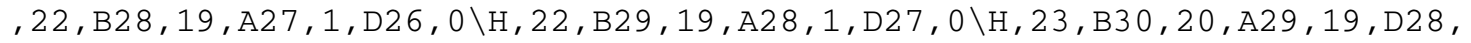
$0 \backslash \mathrm{H}, 23, \mathrm{~B} 31,20, \mathrm{~A} 30,19, \mathrm{D} 29,0 \backslash \mathrm{H}, 23, \mathrm{~B} 32,20, \mathrm{~A} 31,19, \mathrm{D} 30,0 \backslash \mathrm{H}, 24, \mathrm{~B} 33,20, \mathrm{~A} 32,19$

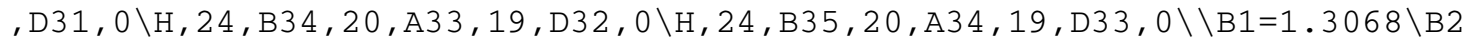
$=1.4102 \backslash \mathrm{B} 3=1.467 \backslash \mathrm{B} 4=1.4261 \backslash \mathrm{B} 5=1.2127 \backslash \mathrm{B} 6=1.2162 \backslash \mathrm{B} 7=1.4279 \backslash \mathrm{B} 8=1.3998 \backslash \mathrm{B} 9=$ $1.3996 \backslash \mathrm{B} 10=1.396 \backslash \mathrm{B} 11=1.3959 \backslash \mathrm{B} 12=1.3975 \backslash \mathrm{B} 13=1.0838 \backslash \mathrm{B} 14=1.0839 \backslash \mathrm{B} 15=1.086$ $7 \backslash \mathrm{B} 16=1.0868 \backslash \mathrm{B} 17=1.0867 \backslash \mathrm{B} 18=1.9095 \backslash \mathrm{B} 19=1.423 \backslash \mathrm{B} 20=1.5167 \backslash \mathrm{B} 21=1.5142 \backslash \mathrm{B} 22$ $=1.4872 \backslash \mathrm{B} 23=1.4998 \backslash \mathrm{B} 24=1.0905 \backslash \mathrm{B} 25=1.0984 \backslash \mathrm{B} 26=1.0937 \backslash \mathrm{B} 27=1.0937 \backslash \mathrm{B} 28=1.0$ $9 \backslash \mathrm{B} 29=1.0992 \backslash \mathrm{B} 30=1.0927 \backslash \mathrm{B} 31=1.0989 \backslash \mathrm{B} 32=1.1121 \backslash \mathrm{B} 33=1.0932 \backslash \mathrm{B} 34=1.0991 \backslash \mathrm{B} 3$ $5=1.1018 \backslash A 1=108.887 \backslash A 2=110.3318 \backslash A 3=107.3011 \backslash A 4=126.6446 \backslash A 5=125.5235 \backslash A 6$ $=125.6869 \backslash \mathrm{A} 7=119.9154 \backslash \mathrm{A} 8=119.5726 \backslash \mathrm{A} 9=119.4327 \backslash \mathrm{A} 10=119.4518 \backslash \mathrm{A} 11=120.480$ $2 \backslash \mathrm{A} 12=119.9913 \backslash \mathrm{A} 13=120.0697 \backslash \mathrm{A} 14=119.3197 \backslash \mathrm{A} 15=119.3048 \backslash \mathrm{A} 16=120.1859 \backslash \mathrm{A} 17$ $=116.0827 \backslash \mathrm{A} 18=94.718 \backslash \mathrm{A} 19=99.3233 \backslash \mathrm{A} 20=103.735 \backslash \mathrm{A} 21=118.9002 \backslash \mathrm{A} 22=121.4914$ $\backslash \mathrm{A} 23=112.9401 \backslash \mathrm{A} 24=108.597 \backslash \mathrm{A} 25=110.2867 \backslash \mathrm{A} 26=109.9282 \backslash \mathrm{A} 27=112.9309 \backslash \mathrm{A} 28=1$ $08.9803 \backslash \mathrm{A} 29=112.3404 \backslash \mathrm{A} 30=112.5638 \backslash \mathrm{A} 31=109.7651 \backslash \mathrm{A} 32=111.1841 \backslash \mathrm{A} 33=111.10$ $7 \backslash \mathrm{A} 34=111.9461 \backslash \mathrm{D} 1=-0.5544 \backslash \mathrm{D} 2=-0.1389 \backslash \mathrm{D} 3=-176.2309 \backslash \mathrm{D} 4=-183.063 \backslash \mathrm{D} 5=183.3$ $039 \backslash D 6=137.2924 \backslash D 7=-42.3188 \backslash D 8=-179.9995 \backslash D 9=179.5194 \backslash D 10=0.446 \backslash D 11=0.6$ $904 \backslash \mathrm{D} 12=0.6929 \backslash \mathrm{D} 13=-179.5752 \backslash \mathrm{D} 14=180.1915 \backslash \mathrm{D} 15=179.8441 \backslash \mathrm{D} 16=-131.2451 \backslash \mathrm{D}$ $17=80.0342 \backslash \mathrm{D} 18=-40.9714 \backslash \mathrm{D} 19=-157.9623 \backslash \mathrm{D} 20=-76.8685 \backslash \mathrm{D} 21=101.5613 \backslash \mathrm{D} 22=54$ $.4977 \backslash \mathrm{D} 23=175.1366 \backslash \mathrm{D} 24=-66.432 \backslash \mathrm{D} 25=58.0462 \backslash \mathrm{D} 26=-62.9506 \backslash \mathrm{D} 27=-183.9468 \backslash$ $\mathrm{D} 28=174.5182 \backslash \mathrm{D} 29=-62.5745 \backslash \mathrm{D} 30=52.9539 \backslash \mathrm{D} 31=177.1667 \backslash \mathrm{D} 32=56.6229 \backslash \mathrm{D} 33=298$ $.5052 \backslash \backslash$ Version=IA64L-G03RevD.01 \State=1 - A \HF=-858.395957 $\backslash$ RMSD $=8.776 e-0$ $9 \backslash \mathrm{RMSF}=5.166 \mathrm{e}-05 \backslash$ ZeroPoint $=0.2909465 \backslash \mathrm{Thermal}=0.3088502 \backslash \mathrm{Dipole}=-0.34779$ 
$67,1.3601922,-1.2479873$

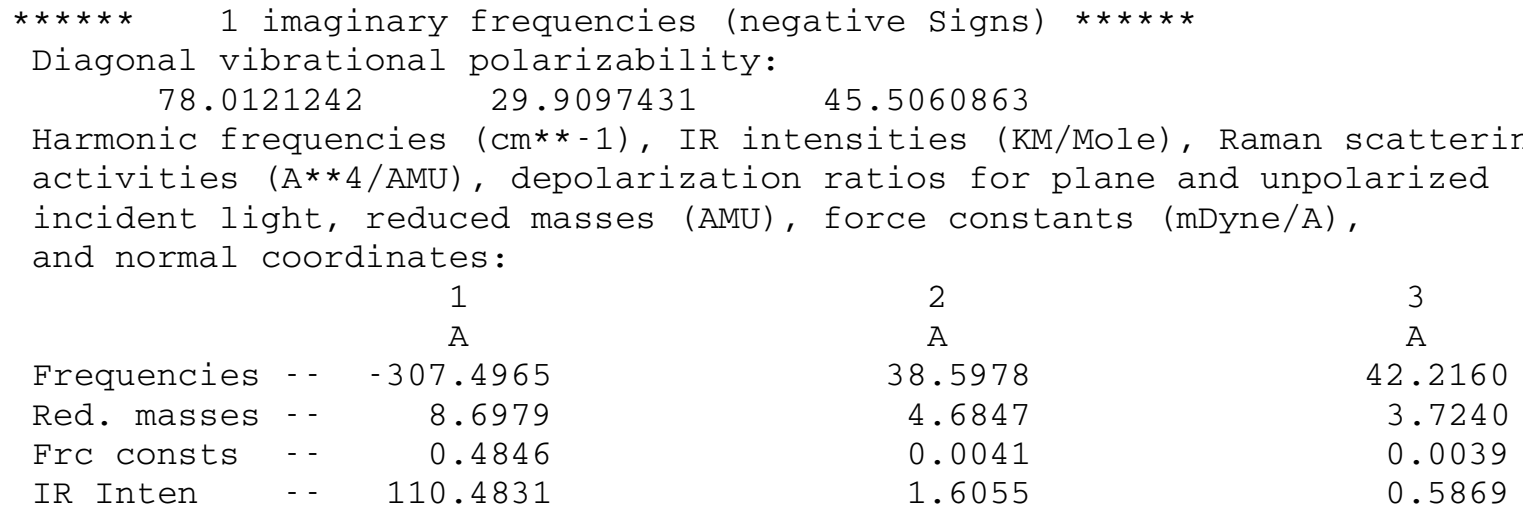

Zero-point correction=

Thermal correction to Energy=

Thermal correction to Enthalpy=

Thermal correction to Gibbs Free Energy=

Sum of electronic and zero-point Energies=

Sum of electronic and thermal Energies=

Sum of electronic and thermal Enthalpies=

Sum of electronic and thermal Free Energies=
$0.290947 \quad$ (Hartree/Particle)
0.308850
0.309794
0.245627
-858.105010
-858.087107
-858.086163
-858.150330

\section{Open Intermediate from B3LYP/6-31+G(d)}

$1 \backslash 1 \backslash$ ASN_ALTIX - ALTIX3 $\backslash$ Freq $\backslash R B 3 L Y P \backslash 6-31+G(d) \backslash C 14 H 17 N 3 O 2 \backslash A U B O X A \backslash 13-A p r-2$

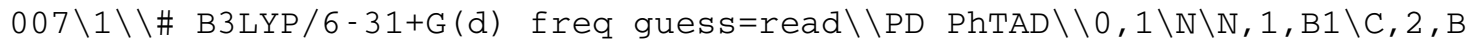
$2,1, \mathrm{~A} 1 \backslash \mathrm{C}, 1, \mathrm{~B} 3,2, \mathrm{~A} 2,3, \mathrm{D} 1,0 \backslash \mathrm{N}, 4, \mathrm{~B} 4,1, \mathrm{~A} 3,2, \mathrm{D} 2,0 \backslash 0,3, \mathrm{~B} 5,2, \mathrm{~A} 4,1, \mathrm{D} 3,0 \backslash 0,4, \mathrm{~B} 6$ $, 1, \mathrm{~A} 5,2, \mathrm{D} 4,0 \backslash \mathrm{C}, 5, \mathrm{~B} 7,4, \mathrm{~A} 6,1, \mathrm{D} 5,0 \backslash \mathrm{C}, 8, \mathrm{~B} 8,5, \mathrm{~A} 7,4, \mathrm{D} 6,0 \backslash \mathrm{C}, 8, \mathrm{~B} 9,5, \mathrm{~A} 8,4, \mathrm{D} 7,0 \backslash$ $\mathrm{C}, 9, \mathrm{~B} 10,8, \mathrm{~A} 9,5, \mathrm{D} 8,0 \backslash \mathrm{C}, 10, \mathrm{~B} 11,8, \mathrm{~A} 10,5, \mathrm{D} 9,0 \backslash \mathrm{C}, 12, \mathrm{~B} 12,10, \mathrm{~A} 11,8, \mathrm{D} 10,0 \backslash \mathrm{H}, 10$ , $\mathrm{B} 13,8, \mathrm{~A} 12,5, \mathrm{D} 11,0 \backslash \mathrm{H}, 9, \mathrm{~B} 14,8, \mathrm{~A} 13,5, \mathrm{D} 12,0 \backslash \mathrm{H}, 12, \mathrm{~B} 15,10, \mathrm{~A} 14,8, \mathrm{D} 13,0 \backslash \mathrm{H}, 11$, $\mathrm{B} 16,9, \mathrm{~A} 15,8, \mathrm{D} 14,0 \backslash \mathrm{H}, 13, \mathrm{~B} 17,12, \mathrm{~A} 16,10, \mathrm{D} 15,0 \backslash \mathrm{C}, 1, \mathrm{~B} 18,4, \mathrm{~A} 17,7, \mathrm{D} 16,0 \backslash \mathrm{C}, 19$, $\mathrm{B} 19,1, \mathrm{~A} 18,4, \mathrm{D} 17,0 \backslash \mathrm{C}, 19, \mathrm{~B} 20,1, \mathrm{~A} 19,4, \mathrm{D} 18,0 \backslash \mathrm{C}, 19, \mathrm{~B} 21,1, \mathrm{~A} 20,4, \mathrm{D} 19,0 \backslash \mathrm{C}, 20, \mathrm{~B}$ $22,19, \mathrm{~A} 21,1, \mathrm{D} 20,0 \backslash \mathrm{C}, 20, \mathrm{~B} 23,19, \mathrm{~A} 22,1, \mathrm{D} 21,0 \backslash \mathrm{H}, 21, \mathrm{~B} 24,19, \mathrm{~A} 23,1, \mathrm{D} 22,0 \backslash \mathrm{H}, 21$ , B25, 19, A24, 1, D23, 0 \H , 21, B26, $19, \mathrm{~A} 25,1, \mathrm{D} 24,0 \backslash \mathrm{H}, 22, \mathrm{~B} 27,19, \mathrm{~A} 26,1, \mathrm{D} 25,0 \backslash \mathrm{H}$, $22, \mathrm{~B} 28,19, \mathrm{~A} 27,1, \mathrm{D} 26,0 \backslash \mathrm{H}, 22, \mathrm{~B} 29,19, \mathrm{~A} 28,1, \mathrm{D} 27,0 \backslash \mathrm{H}, 23, \mathrm{~B} 30,20, \mathrm{~A} 29,19, \mathrm{D} 28,0$ $\backslash \mathrm{H}, 23, \mathrm{~B} 31,20, \mathrm{~A} 30,19, \mathrm{D} 29,0 \backslash \mathrm{H}, 23, \mathrm{~B} 32,20, \mathrm{~A} 31,19, \mathrm{D} 30,0 \backslash \mathrm{H}, 24, \mathrm{~B} 33,20, \mathrm{~A} 32,19$, $\mathrm{D} 31,0 \backslash \mathrm{H}, 24, \mathrm{~B} 34,20, \mathrm{~A} 33,19, \mathrm{D} 32,0 \backslash \mathrm{H}, 24, \mathrm{~B} 35,20, \mathrm{~A} 34,19, \mathrm{D} 33,0 \backslash \backslash \mathrm{B} 1=1.353 \backslash \mathrm{B} 2=1$ $.3751 \backslash B 3=1.4329 \backslash B 4=1.3741 \backslash B 5=1.2197 \backslash B 6=1.2239 \backslash B 7=1.4276 \backslash B 8=1.4003 \backslash B 9=1$ $.4 \backslash B 10=1.396 \backslash B 11=1.396 \backslash B 12=1.3974 \backslash B 13=1.0833 \backslash B 14=1.0838 \backslash B 15=1.0868 \backslash B 16$ $=1.0869 \backslash \mathrm{B} 17=1.0868 \backslash \mathrm{B} 18=1.5047 \backslash \mathrm{B} 19=1.5015 \backslash \mathrm{B} 20=1.5335 \backslash \mathrm{B} 21=1.5319 \backslash \mathrm{B} 22=1.4$ $776 \backslash \mathrm{B} 23=1.4883 \backslash \mathrm{B} 24=1.0891 \backslash \mathrm{B} 25=1.0957 \backslash \mathrm{B} 26=1.0941 \backslash \mathrm{B} 27=1.0947 \backslash \mathrm{B} 28=1.0887 \backslash$ $\mathrm{B} 29=1.096 \backslash \mathrm{B} 30=1.0996 \backslash \mathrm{B} 31=1.1162 \backslash \mathrm{B} 32=1.0926 \backslash \mathrm{B} 33=1.0928 \backslash \mathrm{B} 34=1.0988 \backslash \mathrm{B} 35=1$ $.1057 \backslash A 1=106.5286 \backslash A 2=112.6922 \backslash A 3=103.6096 \backslash A 4=127.5292 \backslash A 5=126.7616 \backslash A 6=1$ $25.1296 \backslash A 7=120.1294 \backslash A 8=119.4878 \backslash A 9=119.5386 \backslash A 10=119.4687 \backslash A 11=120.5622 \backslash$ $\mathrm{A} 12=119.877 \backslash \mathrm{A} 13=120.0602 \backslash \mathrm{A} 14=119.2592 \backslash \mathrm{A} 15=119.3 \backslash \mathrm{A} 16=120.2278 \backslash \mathrm{A} 17=124.9$ $14 \backslash \mathrm{A} 19=109.788 \backslash \mathrm{A} 20=111.6904 \backslash \mathrm{A} 21=118.1558 \backslash \mathrm{A} 22=121.0119 \backslash \mathrm{A} 23=112.9845 \backslash \mathrm{A} 24$ $=108.2841 \backslash \mathrm{A} 25=109.7956 \backslash \mathrm{A} 26=109.2557 \backslash \mathrm{A} 27=113.1304 \backslash \mathrm{A} 28=108.7677 \backslash \mathrm{A} 29=112$. $1594 \backslash \mathrm{A} 30=109.1608 \backslash \mathrm{A} 31=112.395 \backslash \mathrm{A} 32=111.3623 \backslash \mathrm{A} 33=111.2009 \backslash \mathrm{A} 34=111.0887 \backslash \mathrm{D}$ $1=-0.9773 \backslash D 2=1.3236 \backslash D 3=-178.0313 \backslash D 4=-179.9795 \backslash D 5=-183.2211 \backslash D 6=-38.2134$ $\backslash D 7=142.2954 \backslash D 8=180.082 \backslash D 9=-180.6132 \backslash D 10=0.4882 \backslash D 11=0.8563 \backslash D 12=0.8305 \backslash$ $\mathrm{D} 13=180.4627 \backslash \mathrm{D} 14=-179.7607 \backslash \mathrm{D} 15=-180.1766 \backslash \mathrm{D} 16=-19.3418 \backslash \mathrm{D} 17=-67.9391 \backslash \mathrm{D} 18$ $=173.4627 \backslash \mathrm{D} 19=51.9256 \backslash \mathrm{D} 20=-74.7495 \backslash \mathrm{D} 21=99.4968 \backslash \mathrm{D} 22=52.5861 \backslash \mathrm{D} 23=173.939$ $\backslash \mathrm{D} 24=-67.7224 \backslash \mathrm{D} 25=64.9359 \backslash \mathrm{D} 26=-55.656 \backslash \mathrm{D} 27=-177.3553 \backslash \mathrm{D} 28=-66.1748 \backslash \mathrm{D} 29=4$ $8.3674 \backslash \mathrm{D} 30=-189.2883 \backslash \mathrm{D} 31=178.0626 \backslash \mathrm{D} 32=56.2924 \backslash \mathrm{D} 33=-60.5265 \backslash \mathrm{A} 18=92.3323$ 
$\backslash \backslash$ Version=IA64L-G03RevD. 01 \State $=1-A \backslash H F=-858.4071549 \backslash R M S D=9.056 e-09 \backslash R M$ $\mathrm{SF}=8.638 \mathrm{e}-05 \backslash$ ZeroPoint $=0.2930014 \backslash \mathrm{Thermal}=0.3106996 \backslash \mathrm{Dipole}=-1.1969153,1$ $.0510091,-1.7238011$

Zero-point correction=

Thermal correction to Energy=

Thermal correction to Enthalpy=

Thermal correction to Gibbs Free Energy=

Sum of electronic and zero-point Energies=

Sum of electronic and thermal Energies=

Sum of electronic and thermal Enthalpies=

Sum of electronic and thermal Free Energies=

\author{
0.293001 (Hartree/Particle) \\ 0.310700 \\ 0.311644 \\ 0.248394 \\ $-858.114153$ \\ $-858.096455$ \\ $-858.095511$ \\ $-858.158761$
}

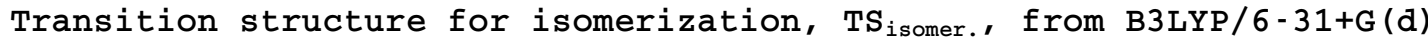

$1 \backslash 1 \backslash A S N \_A L T I X-A L T I X 5 \backslash F r e q \backslash R B 3 L Y P \backslash 6-31+G(d) \backslash C 14 H 17 N 3 O 2 \backslash A U B O X A \backslash 09-M a y-2$

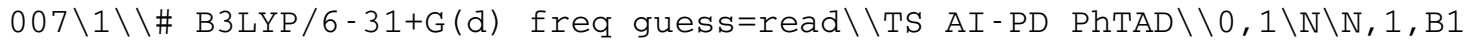
$\backslash \mathrm{C}, 2, \mathrm{~B} 2,1, \mathrm{~A} 1 \backslash \mathrm{C}, 1, \mathrm{~B} 3,2, \mathrm{~A} 2,3, \mathrm{D} 1,0 \backslash \mathrm{N}, 4, \mathrm{~B} 4,1, \mathrm{~A} 3,2, \mathrm{D} 2,0 \backslash 0,3, \mathrm{~B} 5,2, \mathrm{~A} 4,1, \mathrm{D} 3,0 \backslash$ $\mathrm{O}, 4, \mathrm{~B} 6,1, \mathrm{~A} 5,2, \mathrm{D} 4,0 \backslash \mathrm{C}, 5, \mathrm{~B} 7,4, \mathrm{~A} 6,1, \mathrm{D} 5,0 \backslash \mathrm{C}, 8, \mathrm{~B} 8,5, \mathrm{~A} 7,4, \mathrm{D} 6,0 \backslash \mathrm{C}, 8, \mathrm{~B} 9,5, \mathrm{~A} 8,4$

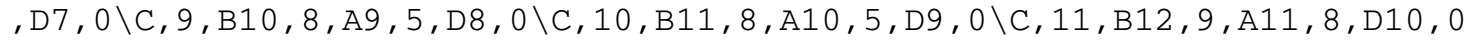
$\backslash \mathrm{H}, 10, \mathrm{~B} 13,8, \mathrm{~A} 12,5, \mathrm{D} 11,0 \backslash \mathrm{H}, 9, \mathrm{~B} 14,8, \mathrm{~A} 13,5, \mathrm{D} 12,0 \backslash \mathrm{H}, 12, \mathrm{~B} 15,10, \mathrm{~A} 14,8, \mathrm{D} 13,0 \backslash$ $\mathrm{H}, 11, \mathrm{~B} 16,9, \mathrm{~A} 15,8, \mathrm{D} 14,0 \backslash \mathrm{H}, 13, \mathrm{~B} 17,11, \mathrm{~A} 16,9, \mathrm{D} 15,0 \backslash \mathrm{C}, 1, \mathrm{~B} 18,2, \mathrm{~A} 17,3, \mathrm{D} 16,0 \backslash \mathrm{C}$

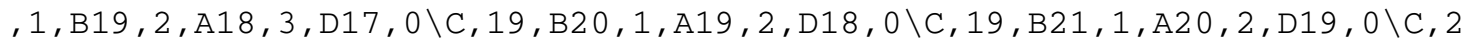
$0, \mathrm{~B} 22,19, \mathrm{~A} 21,1, \mathrm{D} 20,0 \backslash \mathrm{C}, 20, \mathrm{~B} 23,19, \mathrm{~A} 22,1, \mathrm{D} 21,0 \backslash \mathrm{H}, 21, \mathrm{~B} 24,19, \mathrm{~A} 23,1, \mathrm{D} 22,0 \backslash \mathrm{H}$

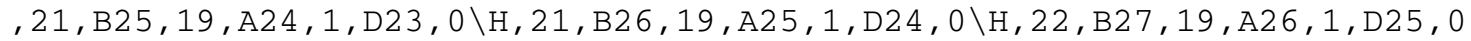
$\backslash \mathrm{H}, 22, \mathrm{~B} 28,19, \mathrm{~A} 27,1, \mathrm{D} 26,0 \backslash \mathrm{H}, 22, \mathrm{~B} 29,19, \mathrm{~A} 28,1, \mathrm{D} 27,0 \backslash \mathrm{H}, 23, \mathrm{~B} 30,20, \mathrm{~A} 29,19, \mathrm{D} 2$ $8,0 \backslash \mathrm{H}, 23, \mathrm{~B} 31,20, \mathrm{~A} 30,19, \mathrm{D} 29,0 \backslash \mathrm{H}, 23, \mathrm{~B} 32,20, \mathrm{~A} 31,19, \mathrm{D} 30,0 \backslash \mathrm{H}, 24, \mathrm{~B} 33,20, \mathrm{~A} 32$, $19, \mathrm{D} 31,0 \backslash \mathrm{H}, 24, \mathrm{~B} 34,20, \mathrm{~A} 33,19, \mathrm{D} 32,0 \backslash \mathrm{H}, 24, \mathrm{~B} 35,20, \mathrm{~A} 34,19, \mathrm{D} 33,0 \backslash \backslash \mathrm{B} 1=1.3746 \backslash$ $\mathrm{B} 2=1.3595 \backslash \mathrm{B} 3=1.4494 \backslash \mathrm{B} 4=1.3636 \backslash \mathrm{B} 5=1.2201 \backslash \mathrm{B} 6=1.2199 \backslash \mathrm{B} 7=1.4274 \backslash \mathrm{B} 8=1.4004 \backslash$ $\mathrm{B} 9=1.4003 \backslash \mathrm{B} 10=1.3962 \backslash \mathrm{B} 11=1.3958 \backslash \mathrm{B} 12=1.3972 \backslash \mathrm{B} 13=1.0832 \backslash \mathrm{B} 14=1.0838 \backslash \mathrm{B} 15=1$ $.0868 \backslash \mathrm{B} 16=1.0869 \backslash \mathrm{B} 17=1.0869 \backslash \mathrm{B} 20=1.5288 \backslash \mathrm{B} 21=1.5287 \backslash \mathrm{B} 22=1.4898 \backslash \mathrm{B} 23=1.498$ $3 \backslash \mathrm{B} 24=1.0882 \backslash \mathrm{B} 25=1.096 \backslash \mathrm{B} 26=1.0942 \backslash \mathrm{B} 27=1.0951 \backslash \mathrm{B} 28=1.0874 \backslash \mathrm{B} 29=1.096 \backslash \mathrm{B} 30=$ $1.0929 \backslash \mathrm{B} 31=1.098 \backslash \mathrm{B} 32=1.1073 \backslash \mathrm{B} 33=1.1023 \backslash \mathrm{B} 34=1.0931 \backslash \mathrm{B} 35=1.094 \backslash \mathrm{A} 1=106.173$ $7 \backslash A 2=112.4264 \backslash A 3=103.2217 \backslash A 4=128.4835 \backslash A 5=126.1014 \backslash A 6=124.9532 \backslash A 7=120.2$ $897 \backslash \mathrm{A} 8=119.3494 \backslash \mathrm{A} 9=119.5529 \backslash \mathrm{A} 10=119.4697 \backslash \mathrm{A} 11=120.4832 \backslash \mathrm{A} 12=119.835 \backslash \mathrm{A} 13=$ $120.0802 \backslash A 14=119.2485 \backslash A 15=119.3028 \backslash A 16=120.2158 \backslash A 17=119.7056 \backslash A 18=115.0$ $407 \backslash \mathrm{A} 19=111.8773 \backslash \mathrm{A} 20=115.168 \backslash \mathrm{A} 21=119.5046 \backslash \mathrm{A} 22=121.56 \backslash \mathrm{A} 23=113.0922 \backslash \mathrm{A} 24=$ $108.2113 \backslash \mathrm{A} 25=109.7154 \backslash \mathrm{A} 26=109.4316 \backslash \mathrm{A} 27=113.838 \backslash \mathrm{A} 28=108.1864 \backslash \mathrm{A} 29=111.39$ $58 \backslash \mathrm{A} 30=111.7537 \backslash \mathrm{A} 31=109.2984 \backslash \mathrm{A} 32=108.5848 \backslash \mathrm{A} 33=113.4441 \backslash \mathrm{A} 34=111.0305 \backslash \mathrm{D} 1$ $=5.2433 \backslash \mathrm{D} 2=-5.1151 \backslash \mathrm{D} 3=178.7253 \backslash \mathrm{D} 4=175.1747 \backslash \mathrm{D} 5=180.8136 \backslash \mathrm{D} 6=-38.3058 \backslash \mathrm{D} 7=$ $142.0492 \backslash \mathrm{D} 8=179.9103 \backslash \mathrm{D} 9=179.5817 \backslash \mathrm{D} 10=0.5755 \backslash \mathrm{D} 11=1.0521 \backslash \mathrm{D} 12=0.6187 \backslash \mathrm{D} 13=$ $-179.5742 \backslash D 14=-179.7484 \backslash D 15=179.6503 \backslash D 16=179.309 \backslash D 17=-124.0432 \backslash D 18=-16$ $.9968 \backslash \mathrm{D} 19=-143.5498 \backslash \mathrm{D} 20=-85.8064 \backslash \mathrm{D} 21=99.8431 \backslash \mathrm{D} 22=45.9404 \backslash \mathrm{D} 23=167.5029 \backslash$ D2 $4=-74.3767 \backslash D 25=69.7934 \backslash D 26=-51.1418 \backslash D 27=187.143 \backslash D 28=-182.453 \backslash D 29=-60$ $.5155 \backslash \mathrm{D} 30=56.6218 \backslash \mathrm{D} 31=81.3745 \backslash \mathrm{D} 32=-37.69 \backslash \mathrm{D} 33=-160.5568 \backslash \mathrm{B} 18=1.4917 \backslash \mathrm{B} 19=$ $1.9302 \backslash \backslash$ Version=IA64L-G03RevD.01 \State=1-A \HF=-858.4056328\RMSD=7.078e $-09 \backslash \mathrm{RMSF}=3.112 \mathrm{e}-05 \backslash$ ZeroPoint $=0.2924567 \backslash \mathrm{Thermal}=0.310051 \backslash \mathrm{Dipole}=-1.4250$ $96,0.7576362,-1.9325737$

$\star * * * * * 1$ imaginary frequencies (negative Signs) $* * * * * *$ Diagonal vibrational polarizability: $50.8297646 \quad 31.6027975 \quad 55.1625963$

Harmonic frequencies ( $\mathrm{cm} * *-1)$, IR intensities (KM/Mole), Raman scattering activities $(A * * 4 / A M U)$, depolarization ratios for plane and unpolarized incident light, reduced masses (AMU), force constants (mDyne/A), and normal coordinates: 
A

$\begin{array}{llr}\text { Frequencies } & - & -182.1485 \\ \text { Red. masses } & -- & 3.9112 \\ \text { Frc consts } & -- & 0.0765 \\ \text { IR Inten } & -- & 7.6983\end{array}$
A

37.6652

4.1210

0.0034

1.9437
A

43.0121

3. 6142

0.0039

0.7777
Zero-point correction=

Thermal correction to Energy=

Thermal correction to Enthalpy=

Thermal correction to Gibbs Free Energy=

Sum of electronic and zero-point Energies=

Sum of electronic and thermal Energies=

Sum of electronic and thermal Enthalpies=

Sum of electronic and thermal Free Energies=

\author{
0.292457 (Hartree/Particle) \\ 0.310051 \\ 0.310995 \\ 0.247542 \\ $-858.113176$ \\ $-858.095582$ \\ $-858.094638$ \\ $-858.158091$
}

\section{AI Intermediate from B3LYP/6-31+G(d)}

$1 \backslash 1 \backslash$ ASN_ALTIX - ALTIX3 \Freq \RB3LYP \6-31+G (d) \C14H17N3O2\AUBOXA \04-Apr - 2

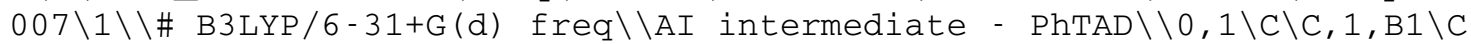
, $2, \mathrm{~B} 2,1, \mathrm{~A} 1 \backslash \mathrm{H}, 3, \mathrm{~B} 3,2, \mathrm{~A} 2,1, \mathrm{D} 1,0 \backslash \mathrm{H}, 3, \mathrm{~B} 4,2, \mathrm{~A} 3,1, \mathrm{D} 2,0 \backslash \mathrm{H}, 3, \mathrm{~B} 5,2, \mathrm{~A} 4,1, \mathrm{D} 3,0 \backslash \mathrm{C}$, $2, \mathrm{~B} 6,1, \mathrm{~A} 5,3, \mathrm{D} 4,0 \backslash \mathrm{H}, 7, \mathrm{~B} 7,2, \mathrm{~A} 6,1, \mathrm{D} 5,0 \backslash \mathrm{H}, 7, \mathrm{~B} 8,2, \mathrm{~A} 7,1, \mathrm{D} 6,0 \backslash \mathrm{H}, 7, \mathrm{~B} 9,2, \mathrm{~A} 8,1, \mathrm{D}$ $7,0 \backslash \mathrm{C}, 1, \mathrm{~B} 10,2, \mathrm{~A} 9,3, \mathrm{D} 8,0 \backslash \mathrm{H}, 11, \mathrm{~B} 11,1, \mathrm{~A} 10,2, \mathrm{D} 9,0 \backslash \mathrm{H}, 11, \mathrm{~B} 12,1, \mathrm{~A} 11,2, \mathrm{D} 10,0 \backslash \mathrm{H}$

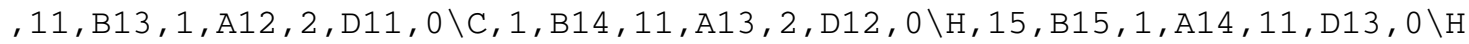

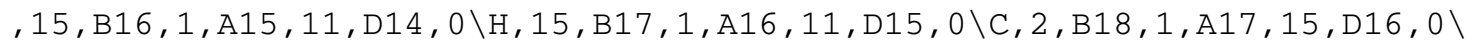
$\mathrm{C}, 19, \mathrm{~B} 19,2, \mathrm{~A} 18,1, \mathrm{D} 17,0 \backslash \mathrm{N}, 20, \mathrm{~B} 20,19, \mathrm{~A} 19,2, \mathrm{D} 18,0 \backslash 0,19, \mathrm{~B} 21,2, \mathrm{~A} 20,1, \mathrm{D} 19,0 \backslash$ $\mathrm{O}, 20, \mathrm{~B} 22,19, \mathrm{~A} 21,2, \mathrm{D} 20,0 \backslash \mathrm{N}, 1, \mathrm{~B} 23,15, \mathrm{~A} 22,11, \mathrm{D} 21,0 \backslash \mathrm{N}, 19, \mathrm{~B} 24,2, \mathrm{~A} 23,1, \mathrm{D} 22,0$ $\backslash \mathrm{C}, 21, \mathrm{~B} 25,20, \mathrm{~A} 24,19, \mathrm{D} 23,0 \backslash \mathrm{C}, 26, \mathrm{~B} 26,21, \mathrm{~A} 25,20, \mathrm{D} 24,0 \backslash \mathrm{C}, 26, \mathrm{~B} 27,21, \mathrm{~A} 26,20$, $\mathrm{D} 25,0 \backslash \mathrm{C}, 27, \mathrm{~B} 28,26, \mathrm{~A} 27,21, \mathrm{D} 26,0 \backslash \mathrm{H}, 27, \mathrm{~B} 29,26, \mathrm{~A} 28,21, \mathrm{D} 27,0 \backslash \mathrm{C}, 28, \mathrm{~B} 30,26, \mathrm{~A} 2$ $9,21, \mathrm{D} 28,0 \backslash \mathrm{H}, 28, \mathrm{~B} 31,26, \mathrm{~A} 30,21, \mathrm{D} 29,0 \backslash \mathrm{C}, 31, \mathrm{~B} 32,28, \mathrm{~A} 31,26, \mathrm{D} 30,0 \backslash \mathrm{H}, 29, \mathrm{~B} 33$, $27, \mathrm{~A} 32,26, \mathrm{D} 31,0 \backslash \mathrm{H}, 31, \mathrm{~B} 34,28, \mathrm{~A} 33,26, \mathrm{D} 32,0 \backslash \mathrm{H}, 33, \mathrm{~B} 35,31, \mathrm{~A} 34,28, \mathrm{D} 33,0 \backslash \backslash \mathrm{B} 1=$ $1.5052 \backslash B 2=1.5193 \backslash B 3=1.0871 \backslash B 4=1.0958 \backslash B 5=1.0969 \backslash B 6=1.5182 \backslash B 7=1.0969 \backslash B 8=$ $1.095 \backslash \mathrm{B} 9=1.0873 \backslash \mathrm{B} 10=1.5186 \backslash \mathrm{B} 11=1.0881 \backslash \mathrm{B} 12=1.0951 \backslash \mathrm{B} 13=1.0968 \backslash \mathrm{B} 14=1.5192$ $\backslash \mathrm{B} 15=1.087 \backslash \mathrm{B} 16=1.097 \backslash \mathrm{B} 17=1.0956 \backslash \mathrm{B} 18=3.5843 \backslash \mathrm{B} 19=2.3322 \backslash \mathrm{B} 20=1.3442 \backslash \mathrm{B} 21=1$ $.2206 \backslash \mathrm{B} 22=1.214 \backslash \mathrm{B} 23=1.5508 \backslash \mathrm{B} 24=1.3378 \backslash \mathrm{B} 25=1.4279 \backslash \mathrm{B} 26=1.4001 \backslash \mathrm{B} 27=1.4 \backslash \mathrm{B} 2$ $8=1.3958 \backslash \mathrm{B} 29=1.0842 \backslash \mathrm{B} 30=1.3958 \backslash \mathrm{B} 31=1.0831 \backslash \mathrm{B} 32=1.3975 \backslash \mathrm{B} 33=1.0869 \backslash \mathrm{B} 34=1$. $0868 \backslash B 35=1.0869 \backslash A 1=121.6719 \backslash A 2=114.3631 \backslash A 3=109.8295 \backslash A 4=107.8365 \backslash A 5=120$ $.5707 \backslash A 6=108.1461 \backslash A 7=109.8682 \backslash A 8=113.3977 \backslash A 9=120.5911 \backslash A 10=112.9429 \backslash A 11$ $=109.8134 \backslash A 12=108.4861 \backslash A 13=112.0249 \backslash A 14=114.6217 \backslash A 15=107.5407 \backslash A 16=109$. $9862 \backslash \mathrm{A} 17=78.0248 \backslash \mathrm{A} 18=48.3235 \backslash \mathrm{A} 19=36.7485 \backslash \mathrm{A} 20=155.8068 \backslash \mathrm{A} 21=168.8911 \backslash \mathrm{A} 22$ $=117.908 \backslash \mathrm{A} 23=31.5082 \backslash \mathrm{A} 24=124.4741 \backslash \mathrm{A} 25=120.2292 \backslash \mathrm{A} 26=119.3039 \backslash \mathrm{A} 27=119.55$ $95 \backslash \mathrm{A} 28=120.103 \backslash \mathrm{A} 29=119.37 \backslash \mathrm{A} 30=119.8062 \backslash \mathrm{A} 31=120.5845 \backslash \mathrm{A} 32=119.3777 \backslash \mathrm{A} 33=1$ $19.2587 \backslash A 34=120.2051 \backslash D 1=29.6584 \backslash D 2=151.3451 \backslash D 3=-91.7237 \backslash D 4=-151.1098 \backslash D$ $5=87.621 \backslash D 6=-154.6738 \backslash D 7=-34.0173 \backslash D 8=151.3049 \backslash D 9=41.8949 \backslash D 10=162.3379 \backslash$ $\mathrm{D} 11=-79.8472 \backslash \mathrm{D} 12=153.8703 \backslash \mathrm{D} 13=-178.1949 \backslash \mathrm{D} 14=-56.8725 \backslash \mathrm{D} 15=59.9 \backslash \mathrm{D} 16=120$. $7165 \backslash \mathrm{D} 17=-78.5035 \backslash \mathrm{D} 18=-164.4957 \backslash \mathrm{D} 19=119.4747 \backslash \mathrm{D} 20=-163.0259 \backslash \mathrm{D} 21=-135.26$ $48 \backslash \mathrm{D} 22=70.3996 \backslash \mathrm{D} 23=-180.8589 \backslash \mathrm{D} 24=-40.6993 \backslash \mathrm{D} 25=139.72 \backslash \mathrm{D} 26=-180.007 \backslash \mathrm{D} 27=$ $0.6864 \backslash \mathrm{D} 28=-180.4219 \backslash \mathrm{D} 29=1.0418 \backslash \mathrm{D} 30=0.3575 \backslash \mathrm{D} 31=180.2404 \backslash \mathrm{D} 32=180.3692 \backslash \mathrm{D}$ $33=-180.1412 \backslash \backslash$ Version=IA64L-G03RevD. $01 \backslash$ State $=1-A \backslash H F=-858.4114643 \backslash R M S D=$ $7.635 e-09 \backslash \mathrm{RMSF}=3.365 \mathrm{e}-05 \backslash$ ZeroPoint $=0.2936687 \backslash$ Thermal $=0.3118722 \backslash \mathrm{Dipole}=$ $1.6242957,-1.8808592,-0.0448897 \backslash$

Zero-point correction=

Thermal correction to Energy=

Thermal correction to Enthalpy=

Thermal correction to Gibbs Free Energy=

Sum of electronic and zero-point Energies= Sum of electronic and thermal Energies=
0.293669 (Hartree/Particle)

0.311872

0.312816

0.247610

$-858.117796$

$-858.099592$ 
Sum of electronic and thermal Enthalpies= Sum of electronic and thermal Free Energies=
$-858.098648$

$-858.163854$

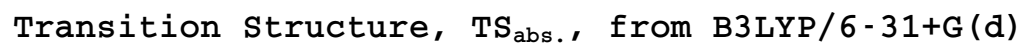

$1 \backslash 1 \backslash A S N \_A L T I X-A L T I X 5 \backslash F r e q \backslash R B 3 L Y P \backslash 6-31+G(d) \backslash C 14 H 17 N 302 \backslash A U B O X A \backslash 07-A p r-2$

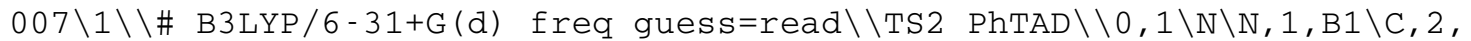
$\mathrm{B} 2,1, \mathrm{~A} 1 \backslash \mathrm{C}, 1, \mathrm{~B} 3,2, \mathrm{~A} 2,3, \mathrm{D} 1,0 \backslash \mathrm{N}, 4, \mathrm{~B} 4,1, \mathrm{~A} 3,2, \mathrm{D} 2,0 \backslash \mathrm{O}, 3, \mathrm{~B} 5,2, \mathrm{~A} 4,1, \mathrm{D} 3,0 \backslash 0,4, \mathrm{~B}$ $6,1, \mathrm{~A} 5,2, \mathrm{D} 4,0 \backslash \mathrm{C}, 5, \mathrm{~B} 7,4, \mathrm{~A} 6,1, \mathrm{D} 5,0 \backslash \mathrm{C}, 8, \mathrm{~B} 8,5, \mathrm{~A} 7,4, \mathrm{D} 6,0 \backslash \mathrm{C}, 8, \mathrm{~B} 9,5, \mathrm{~A} 8,4, \mathrm{D} 7,0$ $\backslash \mathrm{C}, 9, \mathrm{~B} 10,8, \mathrm{~A} 9,5, \mathrm{D} 8,0 \backslash \mathrm{C}, 10, \mathrm{~B} 11,8, \mathrm{~A} 10,5, \mathrm{D} 9,0 \backslash \mathrm{C}, 12, \mathrm{~B} 12,10, \mathrm{~A} 11,8, \mathrm{D} 10,0 \backslash \mathrm{H}, 1$ $0, \mathrm{~B} 13,8, \mathrm{~A} 12,5, \mathrm{D} 11,0 \backslash \mathrm{H}, 9, \mathrm{~B} 14,8, \mathrm{~A} 13,5, \mathrm{D} 12,0 \backslash \mathrm{H}, 12, \mathrm{~B} 15,10, \mathrm{~A} 14,8, \mathrm{D} 13,0 \backslash \mathrm{H}, 11$

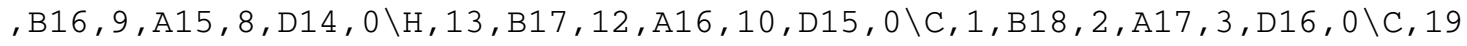

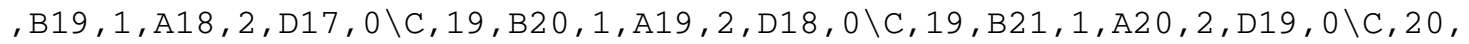
$\mathrm{B} 22,19, \mathrm{~A} 21,1, \mathrm{D} 20,0 \backslash \mathrm{C}, 20, \mathrm{~B} 23,19, \mathrm{~A} 22,1, \mathrm{D} 21,0 \backslash \mathrm{H}, 21, \mathrm{~B} 24,19, \mathrm{~A} 23,1, \mathrm{D} 22,0 \backslash \mathrm{H}, 2$ $1, \mathrm{~B} 25,19, \mathrm{~A} 24,1, \mathrm{D} 23,0 \backslash \mathrm{H}, 21, \mathrm{~B} 26,19, \mathrm{~A} 25,1, \mathrm{D} 24,0 \backslash \mathrm{H}, 22, \mathrm{~B} 27,19, \mathrm{~A} 26,1, \mathrm{D} 25,0 \backslash \mathrm{H}$

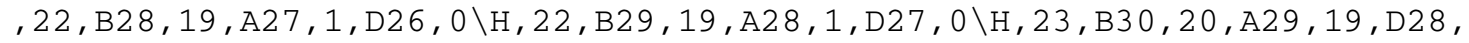
$0 \backslash \mathrm{H}, 23, \mathrm{~B} 31,20, \mathrm{~A} 30,19, \mathrm{D} 29,0 \backslash \mathrm{H}, 23, \mathrm{~B} 32,20, \mathrm{~A} 31,19, \mathrm{D} 30,0 \backslash \mathrm{H}, 24, \mathrm{~B} 33,20, \mathrm{~A} 32,19$ , D31, 0 \H, 24, B34, $20, \mathrm{~A} 33,19, \mathrm{D} 32,0 \backslash \mathrm{H}, 24, \mathrm{~B} 35,20, \mathrm{~A} 34,19, \mathrm{D} 33,0 \backslash \backslash \mathrm{B} 1=1.383 \backslash \mathrm{B} 2=$ $1.3928 \backslash \mathrm{B} 3=1.4174 \backslash \mathrm{B} 4=1.388 \backslash \mathrm{B} 5=1.2163 \backslash \mathrm{B} 6=1.2202 \backslash \mathrm{B} 7=1.4286 \backslash \mathrm{B} 8=1.3998 \backslash \mathrm{B} 9=1$ $.4 \backslash B 10=1.3963 \backslash B 11=1.3956 \backslash B 12=1.3976 \backslash B 13=1.0836 \backslash B 14=1.0835 \backslash B 15=1.0868 \backslash B$ $16=1.0869 \backslash \mathrm{B} 17=1.0868 \backslash \mathrm{B} 18=2.2657 \backslash \mathrm{B} 19=1.5163 \backslash \mathrm{B} 20=1.4143 \backslash \mathrm{B} 21=1.4982 \backslash \mathrm{B} 22=1$ $.5373 \backslash \mathrm{B} 23=1.5328 \backslash \mathrm{B} 24=1.2791 \backslash \mathrm{B} 25=1.0936 \backslash \mathrm{B} 26=1.09 \backslash \mathrm{B} 27=1.0999 \backslash \mathrm{B} 28=1.0937 \backslash$ $\mathrm{B} 29=1.0988 \backslash \mathrm{B} 30=1.0946 \backslash \mathrm{B} 31=1.0954 \backslash \mathrm{B} 32=1.0897 \backslash \mathrm{B} 33=1.0875 \backslash \mathrm{B} 34=1.0943 \backslash \mathrm{B} 35=$ $1.0962 \backslash A 1=106.3375 \backslash A 2=111.3525 \backslash A 3=104.4945 \backslash A 4=126.5403 \backslash A 5=127.2833 \backslash A 6=$ $125.2644 \backslash A 7=120.3171 \backslash A 8=119.3009 \backslash A 9=119.5269 \backslash A 10=119.503 \backslash A 11=120.5229 \backslash$ $\mathrm{A} 12=119.9113 \backslash \mathrm{A} 13=120.0631 \backslash \mathrm{A} 14=119.2857 \backslash \mathrm{A} 15=119.3129 \backslash \mathrm{A} 16=120.2145 \backslash \mathrm{A} 17=9$ $8.0358 \backslash A 18=41.632 \backslash A 19=97.442 \backslash A 20=114.4367 \backslash A 21=115.3949 \backslash A 22=115.6433 \backslash A 2$ $3=98.5866 \backslash A 24=117.8377 \backslash A 25=117.0257 \backslash A 26=109.8865 \backslash A 27=111.1296 \backslash A 28=112$. $5266 \backslash A 29=109.3026 \backslash A 30=108.9403 \backslash A 31=113.2649 \backslash A 32=113.7316 \backslash A 33=109.5078 \backslash$ $\mathrm{A} 34=108.2525 \backslash \mathrm{D} 1=6.8221 \backslash \mathrm{D} 2=-8.2494 \backslash \mathrm{D} 3=177.4832 \backslash \mathrm{D} 4=-186.1358 \backslash \mathrm{D} 5=-174.338$ $3 \backslash D 6=-40.2149 \backslash D 7=139.9503 \backslash D 8=-180.2195 \backslash D 9=179.6414 \backslash D 10=0.5393 \backslash D 11=0.80$ $67 \backslash \mathrm{D} 12=0.8298 \backslash \mathrm{D} 13=180.3616 \backslash \mathrm{D} 14=180.3986 \backslash \mathrm{D} 15=-180.2778 \backslash \mathrm{D} 16=117.8881 \backslash \mathrm{D} 17$ $=120.7537 \backslash \mathrm{D} 18=-1.2816 \backslash \mathrm{D} 19=-130.8328 \backslash \mathrm{D} 20=-113.9206 \backslash \mathrm{D} 21=118.0577 \backslash \mathrm{D} 22=-8$. $2558 \backslash \mathrm{D} 23=90.5883 \backslash \mathrm{D} 24=-129.8468 \backslash \mathrm{D} 25=-120.2076 \backslash \mathrm{D} 26=120.3384 \backslash \mathrm{D} 27=-2.4305 \backslash$ $\mathrm{D} 28=176.0988 \backslash \mathrm{D} 29=-65.5212 \backslash \mathrm{D} 30=56.1793 \backslash \mathrm{D} 31=-48.1969 \backslash \mathrm{D} 32=191.1983 \backslash \mathrm{D} 33=73$

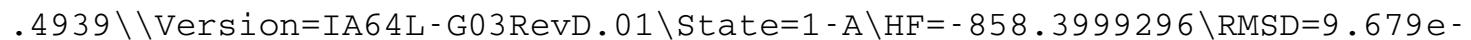
$09 \backslash \mathrm{RMSF}=2.987 \mathrm{e}-05 \backslash$ ZeroPoint $=0.2897989 \backslash \mathrm{Thermal}=0.306794 \backslash \mathrm{Dipole}=-1.12203$ $04,-1.0108653,-1.180748 \backslash$

$\star * * * * * 1$ imaginary frequencies (negative Signs) $* * * * * *$

Diagonal vibrational polarizability: $68.8714072 \quad 26.6023349 \quad 50.1140548$

Harmonic frequencies ( $\mathrm{cm} * *-1)$, IR intensities (KM/Mole), Raman scattering activities $(A * * 4 / A M U)$, depolarization ratios for plane and unpolarized incident light, reduced masses (AMU), force constants (mDyne/A), and normal coordinates:

\begin{tabular}{llr} 
& & \multicolumn{1}{c}{1} \\
& & \multicolumn{1}{c}{ A } \\
Frequencies & -- & -990.0455 \\
Red. masses & -- & 1.2722 \\
Frc consts & -- & 0.7347 \\
IR Inten & -- & 316.8513
\end{tabular}

A
37.8760
4.6263
0.0039
2.0831

Zero-point correction=

Thermal correction to Energy=

Thermal correction to Enthalpy=

Thermal correction to Gibbs Free Energy=

3
A
42.0420
3.7481
0.0039
0.9627

0.289799 (Hartree/Particle)

0.306794

0.307738

0.245755 
Sum of electronic and zero-point Energies= Sum of electronic and thermal Energies= Sum of electronic and thermal Enthalpies= Sum of electronic and thermal Free Energies=
$-858.110131$

$-858.093136$

$-858.092191$

$-858.154174$

\section{Ene product from B3LYP/6-31+G(d)}

$1 \backslash 1 \backslash$ ASN_ALTIX - ALTIX2 \Freq $\backslash R B 3 L Y P \backslash 6-31+G(d) \backslash C 14 H 17 N 302 \backslash A U B O X A \backslash 06-M a y-2$

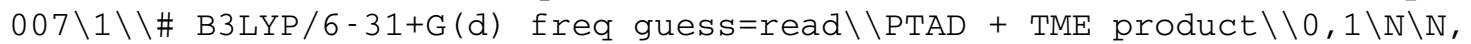
1, $\mathrm{B} 1 \backslash \mathrm{C}, 2, \mathrm{~B} 2,1, \mathrm{~A} 1 \backslash \mathrm{C}, 1, \mathrm{~B} 3,2, \mathrm{~A} 2,3, \mathrm{D} 1,0 \backslash \mathrm{N}, 4, \mathrm{~B} 4,1, \mathrm{~A} 3,2, \mathrm{D} 2,0 \backslash 0,3, \mathrm{~B} 5,2, \mathrm{~A} 4,1, \mathrm{D}$ $3,0 \backslash 0,4, \mathrm{~B} 6,1, \mathrm{~A} 5,2, \mathrm{D} 4,0 \backslash \mathrm{C}, 5, \mathrm{~B} 7,4, \mathrm{~A} 6,1, \mathrm{D} 5,0 \backslash \mathrm{C}, 8, \mathrm{~B} 8,5, \mathrm{~A} 7,4, \mathrm{D} 6,0 \backslash \mathrm{C}, 8, \mathrm{~B} 9,5$, $\mathrm{A} 8,4, \mathrm{D} 7,0 \backslash \mathrm{C}, 9, \mathrm{~B} 10,8, \mathrm{~A} 9,5, \mathrm{D} 8,0 \backslash \mathrm{C}, 10, \mathrm{~B} 11,8, \mathrm{~A} 10,5, \mathrm{D} 9,0 \backslash \mathrm{C}, 12, \mathrm{~B} 12,10, \mathrm{~A} 11,8$, $\mathrm{D} 10,0 \backslash \mathrm{H}, 10, \mathrm{~B} 13,8, \mathrm{~A} 12,5, \mathrm{D} 11,0 \backslash \mathrm{H}, 9, \mathrm{~B} 14,8, \mathrm{~A} 13,5, \mathrm{D} 12,0 \backslash \mathrm{H}, 12, \mathrm{~B} 15,10, \mathrm{~A} 14,8, \mathrm{D}$ $13,0 \backslash \mathrm{H}, 11, \mathrm{~B} 16,9, \mathrm{~A} 15,8, \mathrm{D} 14,0 \backslash \mathrm{H}, 13, \mathrm{~B} 17,12, \mathrm{~A} 16,10, \mathrm{D} 15,0 \backslash \mathrm{C}, 1, \mathrm{~B} 18,4, \mathrm{~A} 17,7, \mathrm{D}$ $16,0 \backslash \mathrm{C}, 19, \mathrm{~B} 19,1, \mathrm{~A} 18,4, \mathrm{D} 17,0 \backslash \mathrm{C}, 19, \mathrm{~B} 20,1, \mathrm{~A} 19,4, \mathrm{D} 18,0 \backslash \mathrm{C}, 19, \mathrm{~B} 21,1, \mathrm{~A} 20,4, \mathrm{D} 1$ $9,0 \backslash \mathrm{C}, 20, \mathrm{~B} 22,19, \mathrm{~A} 21,1, \mathrm{D} 20,0 \backslash \mathrm{C}, 20, \mathrm{~B} 23,19, \mathrm{~A} 22,1, \mathrm{D} 21,0 \backslash \mathrm{H}, 2, \mathrm{~B} 24,1, \mathrm{~A} 23,4, \mathrm{D} 2$ $2,0 \backslash \mathrm{H}, 21, \mathrm{~B} 25,19, \mathrm{~A} 24,1, \mathrm{D} 23,0 \backslash \mathrm{H}, 21, \mathrm{~B} 26,19, \mathrm{~A} 25,1, \mathrm{D} 24,0 \backslash \mathrm{H}, 22, \mathrm{~B} 27,19, \mathrm{~A} 26,1$, $\mathrm{D} 25,0 \backslash \mathrm{H}, 22, \mathrm{~B} 28,19, \mathrm{~A} 27,1, \mathrm{D} 26,0 \backslash \mathrm{H}, 22, \mathrm{~B} 29,19, \mathrm{~A} 28,1, \mathrm{D} 27,0 \backslash \mathrm{H}, 23, \mathrm{~B} 30,20, \mathrm{~A} 29$, $19, \mathrm{D} 28,0 \backslash \mathrm{H}, 23, \mathrm{~B} 31,20, \mathrm{~A} 30,19, \mathrm{D} 29,0 \backslash \mathrm{H}, 23, \mathrm{~B} 32,20, \mathrm{~A} 31,19, \mathrm{D} 30,0 \backslash \mathrm{H}, 24, \mathrm{~B} 33,20$ , A32, 19, D31, 0\H , $24, \mathrm{~B} 34,20, \mathrm{~A} 33,19, \mathrm{D} 32,0 \backslash \mathrm{H}, 24, \mathrm{~B} 35,20, \mathrm{~A} 34,19, \mathrm{D} 33,0 \backslash \backslash \mathrm{B} 1=1$. $4251 \backslash B 2=1.3915 \backslash B 3=1.3932 \backslash B 4=1.4111 \backslash B 5=1.2163 \backslash B 6=1.2177 \backslash B 7=1.4293 \backslash B 8=1$. $3991 \backslash \mathrm{B} 9=1.3992 \backslash \mathrm{B} 10=1.3959 \backslash \mathrm{B} 11=1.3959 \backslash \mathrm{B} 12=1.3974 \backslash \mathrm{B} 13=1.0841 \backslash \mathrm{B} 14=1.0837 \backslash$ $\mathrm{B} 15=1.0867 \backslash \mathrm{B} 16=1.0867 \backslash \mathrm{B} 17=1.0868 \backslash \mathrm{B} 18=2.4276 \backslash \mathrm{B} 19=1.5405 \backslash \mathrm{B} 20=1.3392 \backslash \mathrm{B} 21=$ $1.5151 \backslash \mathrm{B} 22=1.5373 \backslash \mathrm{B} 23=1.5447 \backslash \mathrm{B} 24=1.0157 \backslash \mathrm{B} 25=1.0847 \backslash \mathrm{B} 26=1.0877 \backslash \mathrm{B} 27=1.09$ $43 \backslash B 28=1.097 \backslash B 29=1.0972 \backslash B 30=1.095 \backslash B 31=1.0948 \backslash B 32=1.0938 \backslash B 33=1.09 \backslash B 34=1$ $.0957 \backslash B 35=1.0929 \backslash A 1=108.9263 \backslash A 2=106.8624 \backslash A 3=106.1509 \backslash A 4=126.1572 \backslash A 5=12$ $7.6176 \backslash A 6=124.656 \backslash A 7=119.9199 \backslash A 8=119.5433 \backslash A 9=119.4298 \backslash A 10=119.4587 \backslash A 11$ $=120.4511 \backslash \mathrm{A} 12=120.0309 \backslash \mathrm{A} 13=120.0595 \backslash \mathrm{A} 14=119.331 \backslash \mathrm{A} 15=119.3137 \backslash \mathrm{A} 16=120.1$ $799 \backslash \mathrm{A} 17=159.7962 \backslash \mathrm{A} 18=36.6959 \backslash \mathrm{A} 19=132.1091 \backslash \mathrm{A} 20=96.7101 \backslash \mathrm{A} 21=114.1186 \backslash \mathrm{A} 22$ $=108.7258 \backslash \mathrm{A} 23=114.1846 \backslash \mathrm{A} 24=123.1208 \backslash \mathrm{A} 25=120.7538 \backslash \mathrm{A} 26=110.7856 \backslash \mathrm{A} 27=111$. $8106 \backslash \mathrm{A} 28=111.4103 \backslash \mathrm{A} 29=109.3119 \backslash \mathrm{A} 30=110.6301 \backslash \mathrm{A} 31=111.7648 \backslash \mathrm{A} 32=111.236 \backslash \mathrm{A}$ $33=108.243 \backslash A 34=111.7851 \backslash D 1=-15.8913 \backslash D 2=11.9041 \backslash D 3=-167.046 \backslash D 4=191.8021$ $\backslash \mathrm{D} 5=-184.573 \backslash \mathrm{D} 6=-41.0706 \backslash \mathrm{D} 7=139.1444 \backslash \mathrm{D} 8=-180.1218 \backslash \mathrm{D} 9=179.4785 \backslash \mathrm{D} 10=0.64$ $77 \backslash \mathrm{D} 11=0.5537 \backslash \mathrm{D} 12=0.9006 \backslash \mathrm{D} 13=-179.565 \backslash \mathrm{D} 14=-179.6026 \backslash \mathrm{D} 15=-180.3069 \backslash \mathrm{D} 16=$ $-12.6755 \backslash D 17=-26.066 \backslash D 18=243.8041 \backslash D 19=100.6729 \backslash D 20=-120.0239 \backslash D 21=117.2$ $264 \backslash D 22=-146.6713 \backslash D 23=44.3028 \backslash D 24=-136.2611 \backslash D 25=136.7937 \backslash D 26=15.6193 \backslash D$ $27=-103.4035 \backslash \mathrm{D} 28=179.4894 \backslash \mathrm{D} 29=-61.9623 \backslash \mathrm{D} 30=59.4672 \backslash \mathrm{D} 31=172.6792 \backslash \mathrm{D} 32=52$ $.7919 \backslash D 33=-66.7917 \backslash \backslash V e r s i o n=I A 64 L-G 03$ RevD.01 $\backslash$ State $=1-A \backslash H F=-858.4554189$ $\backslash \mathrm{RMSD}=9.551 \mathrm{e}-09 \backslash \mathrm{RMSF}=2.833 \mathrm{e}-05 \backslash$ ZeroPoint $=0.295502 \backslash \mathrm{Thermal}=0.3132779 \backslash \mathrm{Di}$ pole $=-1.0187758,-0.1475199,-0.4207241$

Zero-point correction=

Thermal correction to Energy=

Thermal correction to Enthalpy=

Thermal correction to Gibbs Free Energy=

Sum of electronic and zero-point Energies=

Sum of electronic and thermal Energies=

Sum of electronic and thermal Enthalpies=

Sum of electronic and thermal Free Energies=

\author{
0.295502 (Hartree/Particle) \\ 0.313278 \\ 0.314222 \\ 0.249610 \\ $-858.159917$ \\ $-858.142141$ \\ $-858.141197$ \\ $-858.205809$
}

Tetramethylethylene in water from B3LYP/6-311++G $(2 d, p) / / B 3 L Y P / 6-31+G(d)$ and CPCM

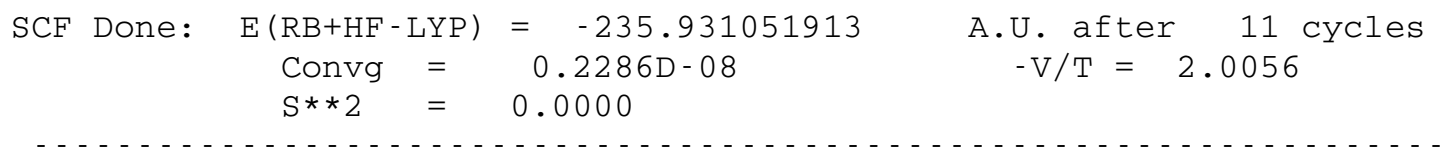

Variational C-PCM results

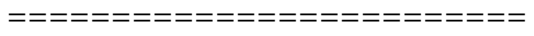




\begin{tabular}{|c|c|c|c|}
\hline$<\operatorname{psi}(\mathrm{f})|\quad \mathrm{H} \quad| \operatorname{psi}(\mathrm{f})>$ & $(\mathrm{a} \cdot \mathrm{u})$. & & -235.929333 \\
\hline$<\operatorname{psi}(\mathrm{f})|\mathrm{H}+\mathrm{V}(\mathrm{f}) / 2| \mathrm{psi}(\mathrm{f})>$ & $(\mathrm{a} \cdot \mathrm{u})$. & $=$ & -235.931052 \\
\hline Total free energy in solution: & & & \\
\hline with all non electrostatic terms & $(\mathrm{a} \cdot \mathrm{u} \cdot)$ & $=$ & -235.917132 \\
\hline - - - - - - - - - - - - - - & 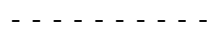 & -- & $-\ldots$ \\
\hline (Polarized solute)-Solvent & $(\mathrm{kcal} / \mathrm{mol})$ & $=$ & -1.08 \\
\hline - - - - - - - & $\ldots \ldots$ & -- & 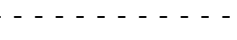 \\
\hline Cavitation energy & $(\mathrm{kcal} / \mathrm{mol})$ & $=$ & 21.21 \\
\hline Dispersion energy & $(\mathrm{kcal} / \mathrm{mol})$ & $=$ & -13.36 \\
\hline Repulsion energy & $(\mathrm{kcal} / \mathrm{mol})$ & $=$ & 0.89 \\
\hline Total non electrostatic & $(\mathrm{kcal} / \mathrm{mol})$ & $=$ & 8.74 \\
\hline
\end{tabular}

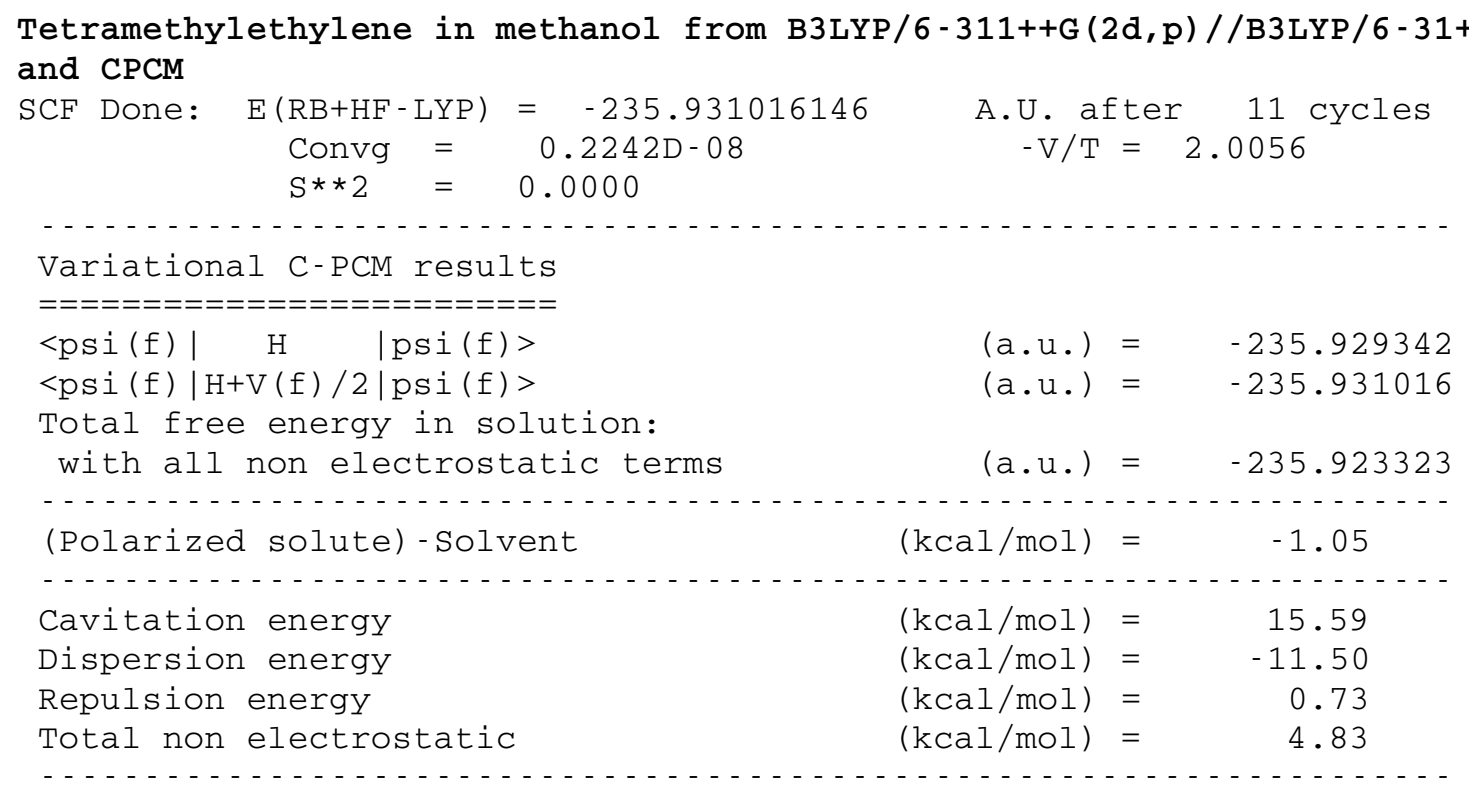

Tetramethylethylene in dmso from B3LYP/6-311++G(2d,p)//B3LYP/6-31+G(d) and CPCM

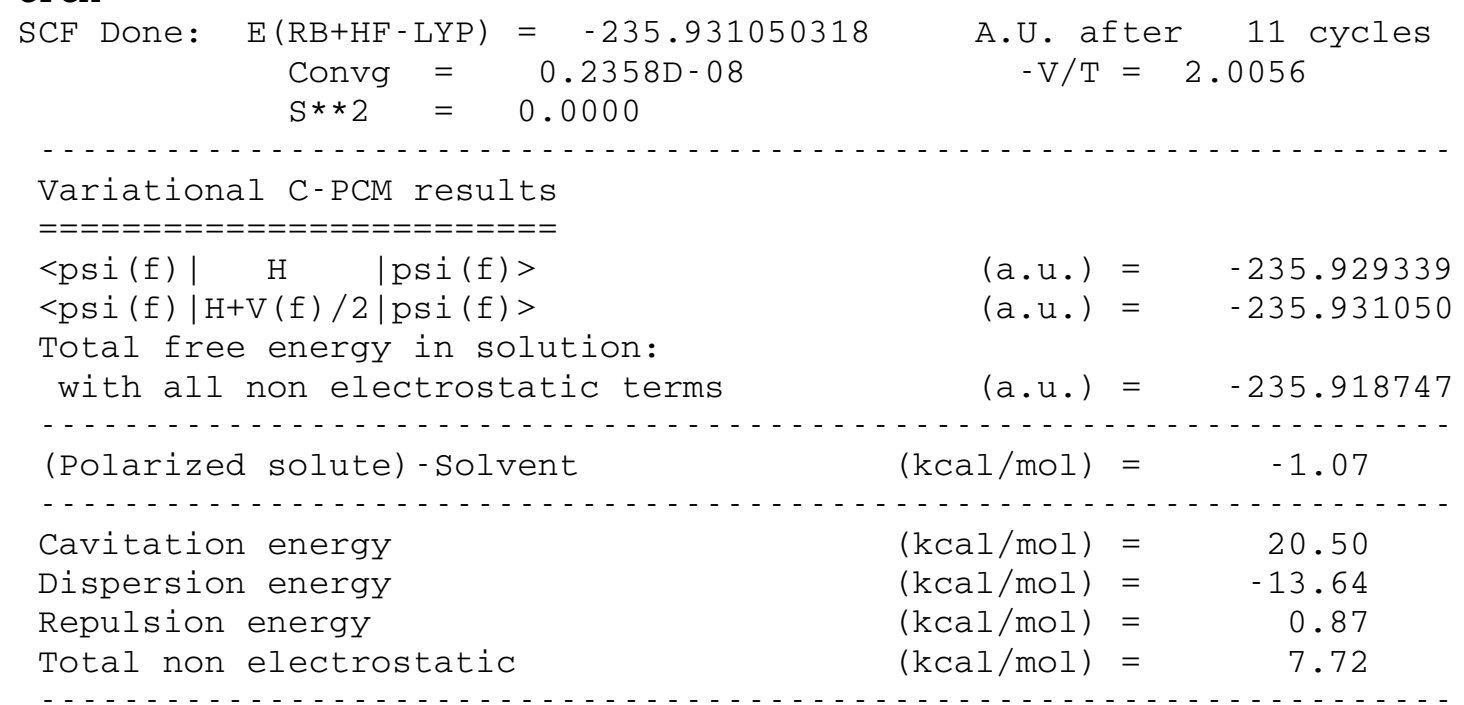

Tetramethylethylene in acetonitrile from B3LYP/6-311++G(2d,p)//B3LYP/6$31+\mathrm{G}(\mathrm{d})$ and $\mathrm{CPCM}$ 


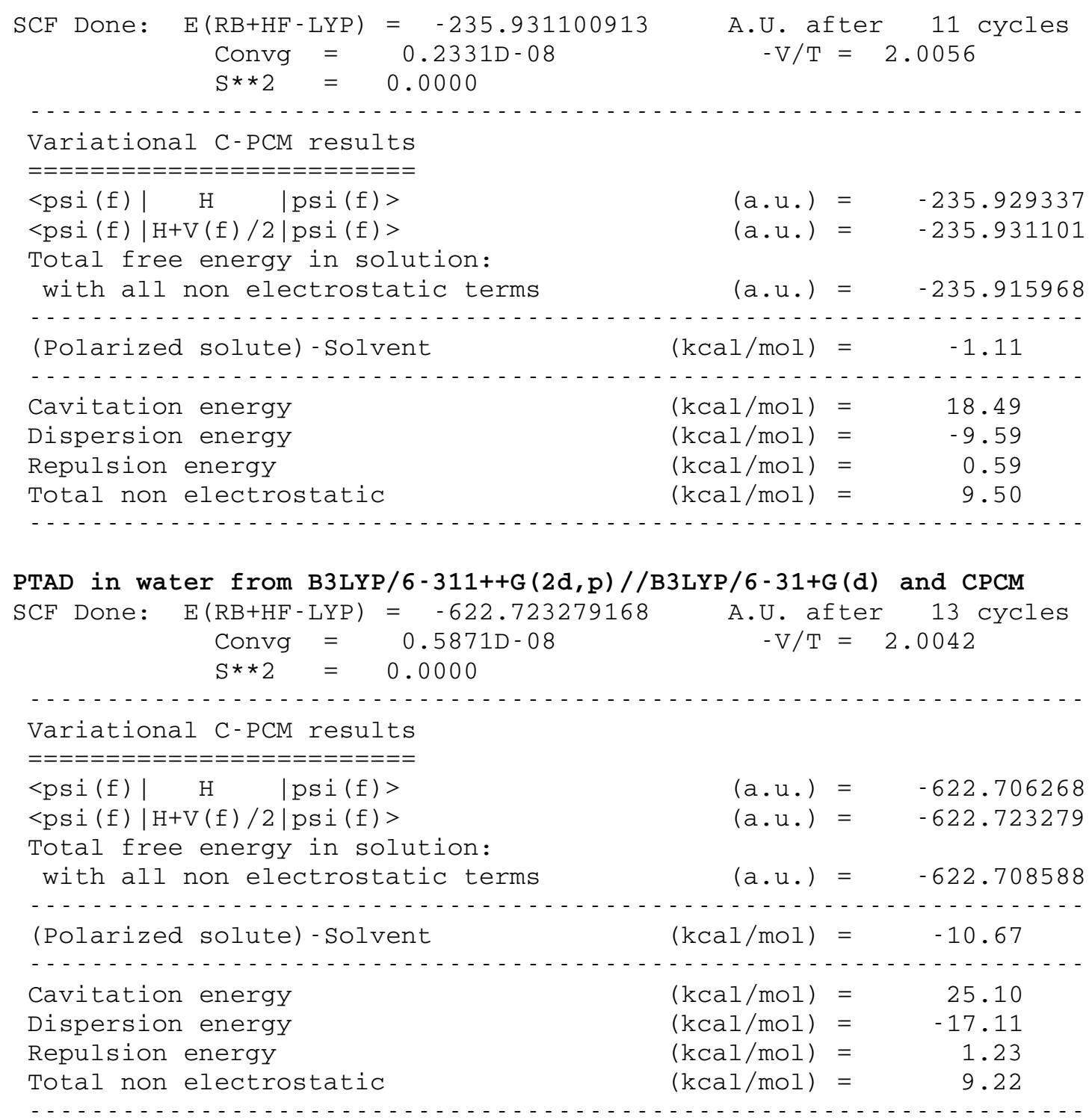

PTAD in methanol from B3LYP/6-311++G (2d,p)//B3LYP/6-31+G(d) and CPCM SCF Done: $\mathrm{E}(\mathrm{RB}+\mathrm{HF}-\mathrm{LYP})=-622.722620112$ A.U. after 13 cycles Convg $=0.6051 \mathrm{D}-08 \quad-\mathrm{V} / \mathrm{T}=2.0042$ $\mathrm{S} * * 2=0.0000$

Variational C-PCM results

$===================$

$<\operatorname{psi}(f)|\quad H \quad| \operatorname{psi}(f)>\quad$ (a.u.) $=\quad-622.706440$

<psi(f) $|\mathrm{H}+\mathrm{V}(\mathrm{f}) / 2| \mathrm{psi}(\mathrm{f})>\quad$ (a.u.) $=\quad-622.722620$

Total free energy in solution:

with all non electrostatic terms (a.u.) = $\quad-622.715391$

- . . . . . . . . . . . . . . . . . . . . . . . . . . . . . . . .

(Polarized solute)-Solvent $\quad(\mathrm{kcal} / \mathrm{mol})=-10.15$

Cavitation energy

Dispersion energy

$(\mathrm{kcal} / \mathrm{mol})=18.25$

$(\mathrm{kcal} / \mathrm{mol})=-14.74$

Repulsion energy

Total non electrostatic

$(\mathrm{kcal} / \mathrm{mol})=1.02$

$(\mathrm{kcal} / \mathrm{mol})=4.54$ 


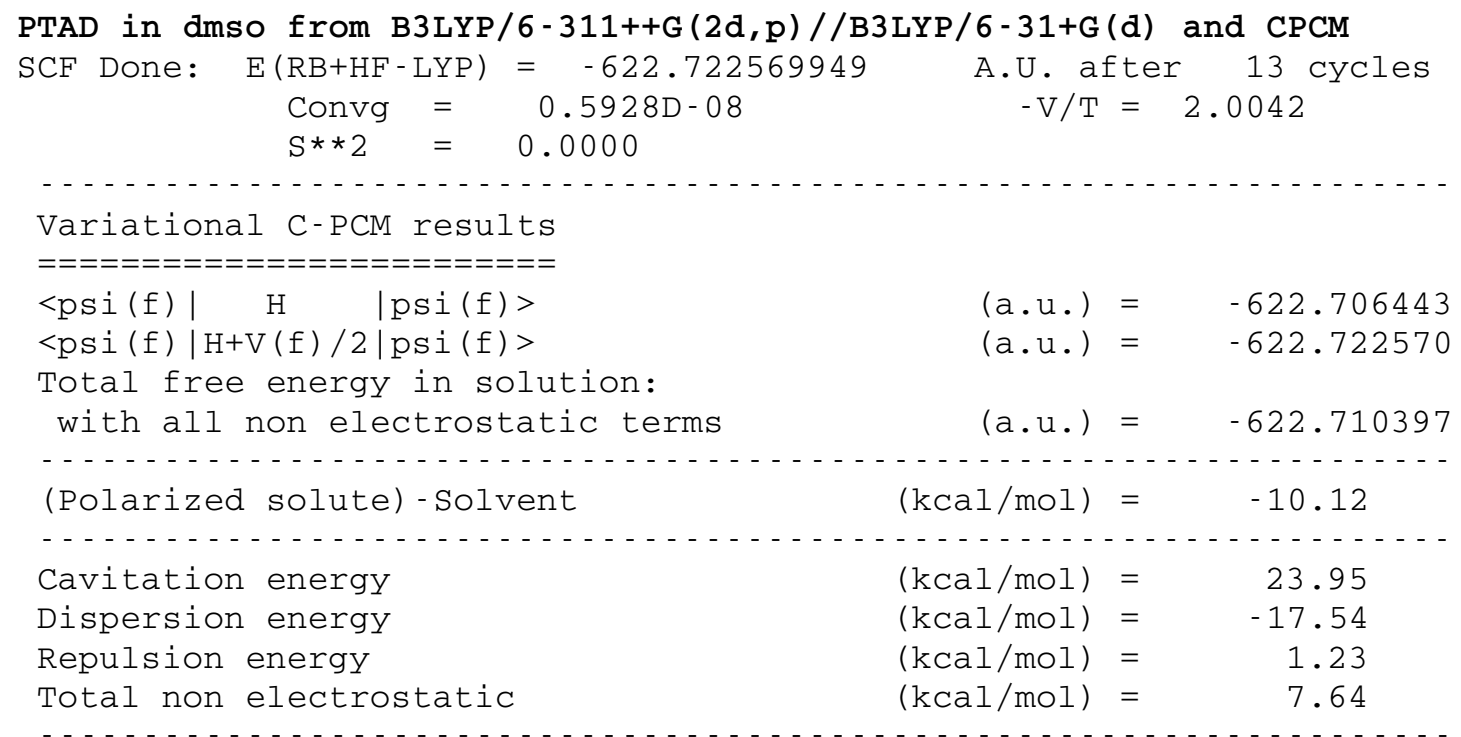

PTAD in acetonitrile from B3LYP/6-311++G(2d,p)//B3LYP/6-31+G(d) and CPCM

$\begin{array}{cccc}\mathrm{SCF} \text { Done: } & \begin{array}{c}\mathrm{E}(\mathrm{RB}+\mathrm{HF}-\mathrm{LYP}) \\ \text { Convg }\end{array} & -622.722589952 & \mathrm{~A} . \mathrm{U} \text {. after } 13 \text { cycles } \\ \mathrm{S} * 2 & 0.5874 \mathrm{D}-08 & -\mathrm{V} / \mathrm{T}=2.0042\end{array}$

Variational C-PCM results

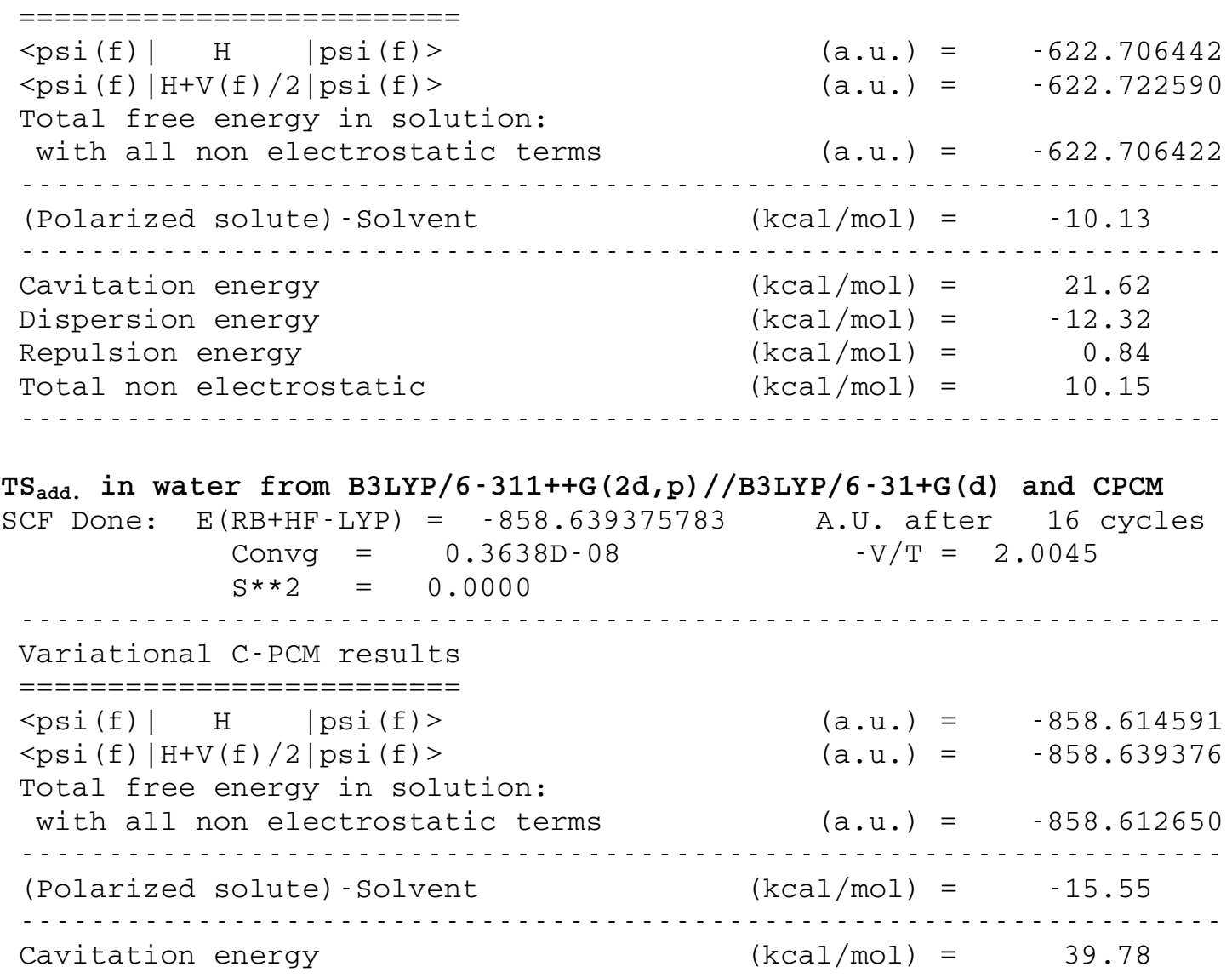




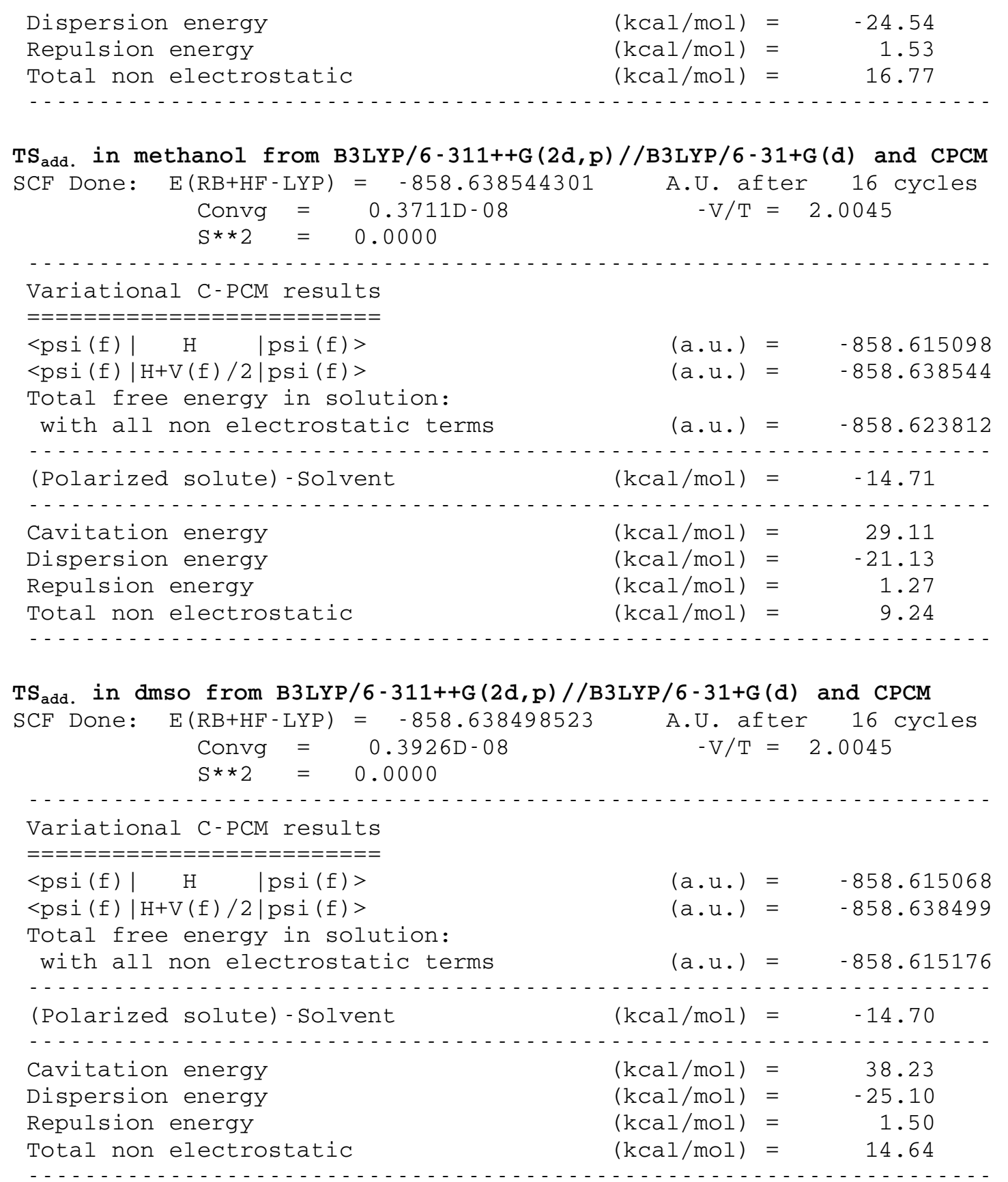

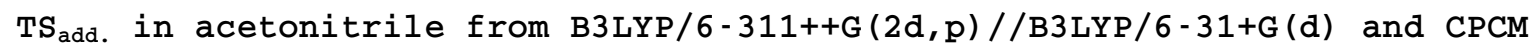
$\mathrm{SCF}$ Done: $\mathrm{E}(\mathrm{RB}+\mathrm{HF}-\mathrm{LYP})=-858.638454575$ A.U. after 16 cycles

Convg $=0.3910 \mathrm{D}-08 \quad-\mathrm{V} / \mathrm{T}=2.0045$
$\mathrm{S} * * 2=0.0000$

Variational C-PCM results

$====================$

$<\operatorname{psi}(f)|\quad \mathrm{H} \quad| \operatorname{psi}(\mathrm{f})>\quad$ (a.u.) $=-858.615139$

<psi(f) $|\mathrm{H}+\mathrm{V}(\mathrm{f}) / 2| \mathrm{psi}(\mathrm{f})>\quad$ (a.u.) $=\quad-858.638455$

Total free energy in solution:

with all non electrostatic terms (a.u.) = -858.609929 


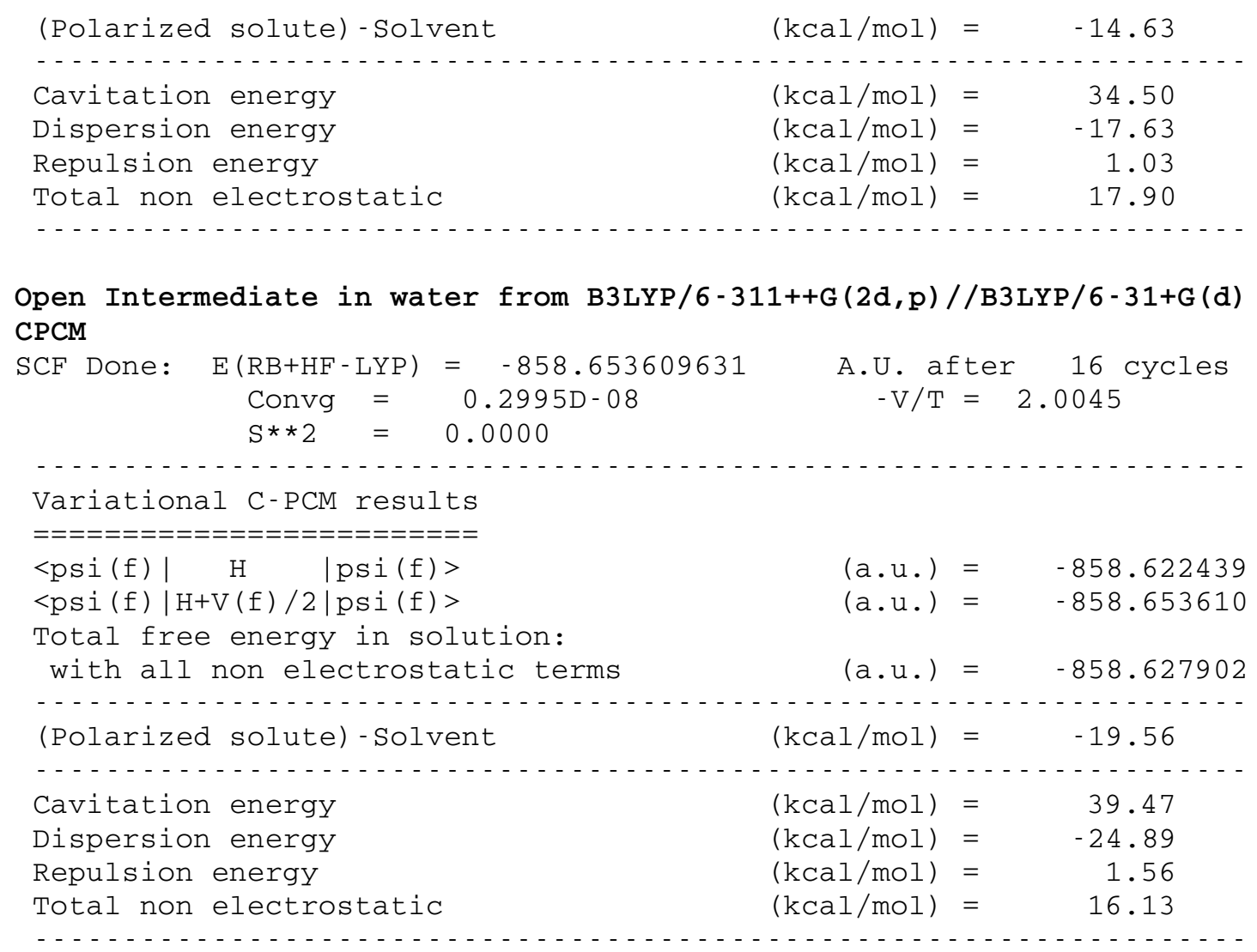

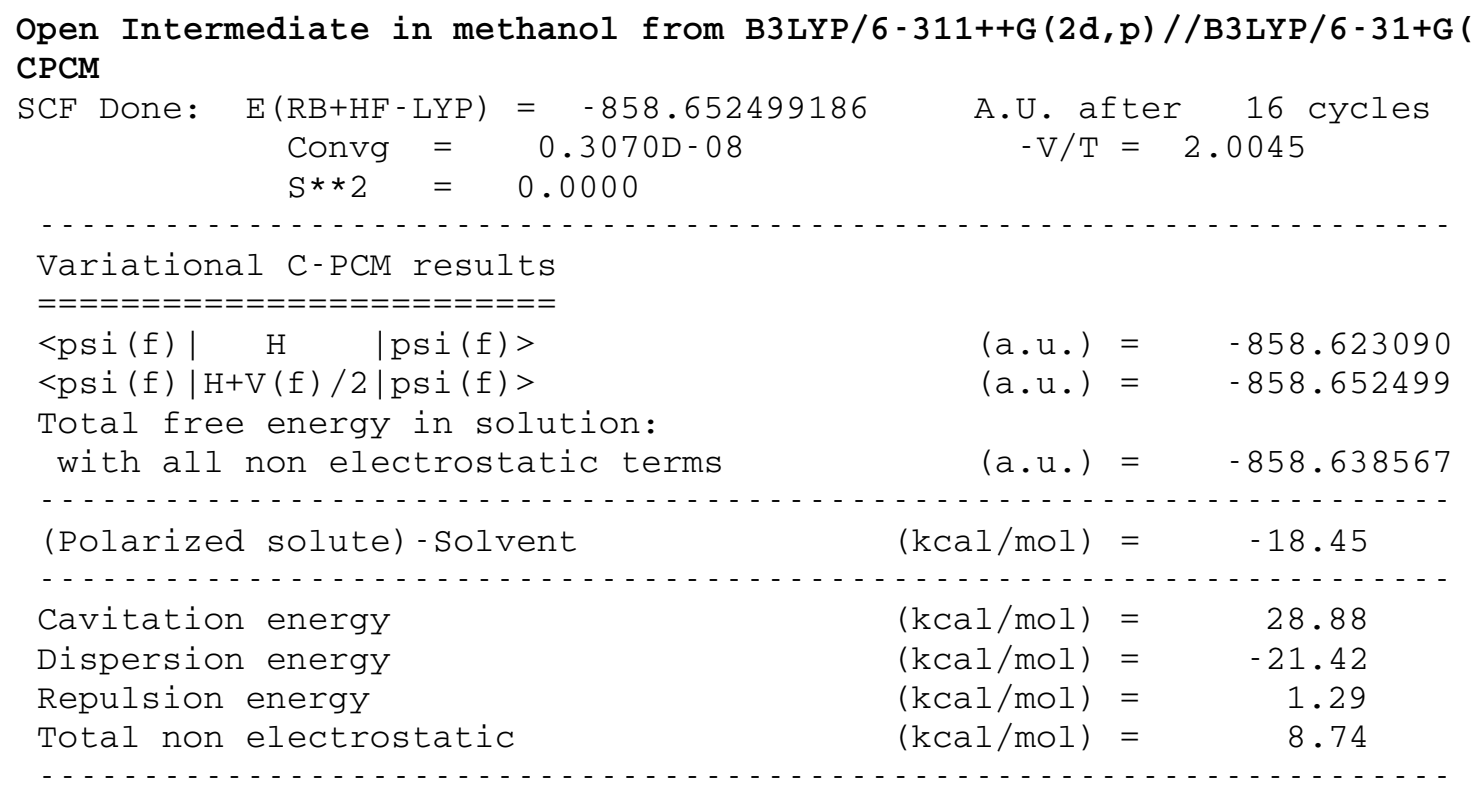

Open Intermediate in dmso from B3LYP/6-311++G (2d,p)//B3LYP/6-31+G(d) and CPCM $\mathrm{SCF}$ Done: $\mathrm{E}(\mathrm{RB}+\mathrm{HF}-\mathrm{LYP})=-858.652484568 \quad$ A.U. after 16 cycles

Convg $=0.3117 \mathrm{D}-08 \quad-\mathrm{V} / \mathrm{T}=2.0045$

$\mathrm{S} * * 2=0.0000$

Variational C-PCM results

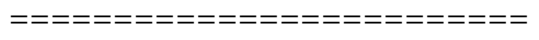




\begin{tabular}{|c|c|c|c|}
\hline$<\operatorname{psi}(f)|\quad H \quad| \operatorname{psi}(f)>$ & $(\mathrm{a} \cdot \mathrm{u})$. & & -858.623010 \\
\hline$<p s i(f)|H+V(f) / 2| p s i(f)>$ & $(\mathrm{a} \cdot \mathrm{u})$. & $=$ & -858.652485 \\
\hline Total free energy in solution: & & & \\
\hline with all non electrostatic terms & $(\mathrm{a} \cdot \mathrm{u} \cdot)$ & $=$ & -858.630122 \\
\hline - - - - - - - - - - - - - - - - - - - - & $\ldots$ & - & $\ldots \ldots \ldots$ \\
\hline (Polarized solute)-Solvent & $(\mathrm{kcal} / \mathrm{mol})$ & $=$ & -18.50 \\
\hline - - - - - - - & $\ldots \ldots$ & -- & 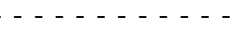 \\
\hline Cavitation energy & $(\mathrm{kcal} / \mathrm{mol})$ & $=$ & 37.93 \\
\hline Dispersion energy & $(\mathrm{kcal} / \mathrm{mol})$ & $=$ & -25.42 \\
\hline Repulsion energy & $(\mathrm{kcal} / \mathrm{mol})$ & $=$ & 1.52 \\
\hline Total non electrostatic & ( $\mathrm{kcal} / \mathrm{mol})$ & $=$ & 14.03 \\
\hline
\end{tabular}

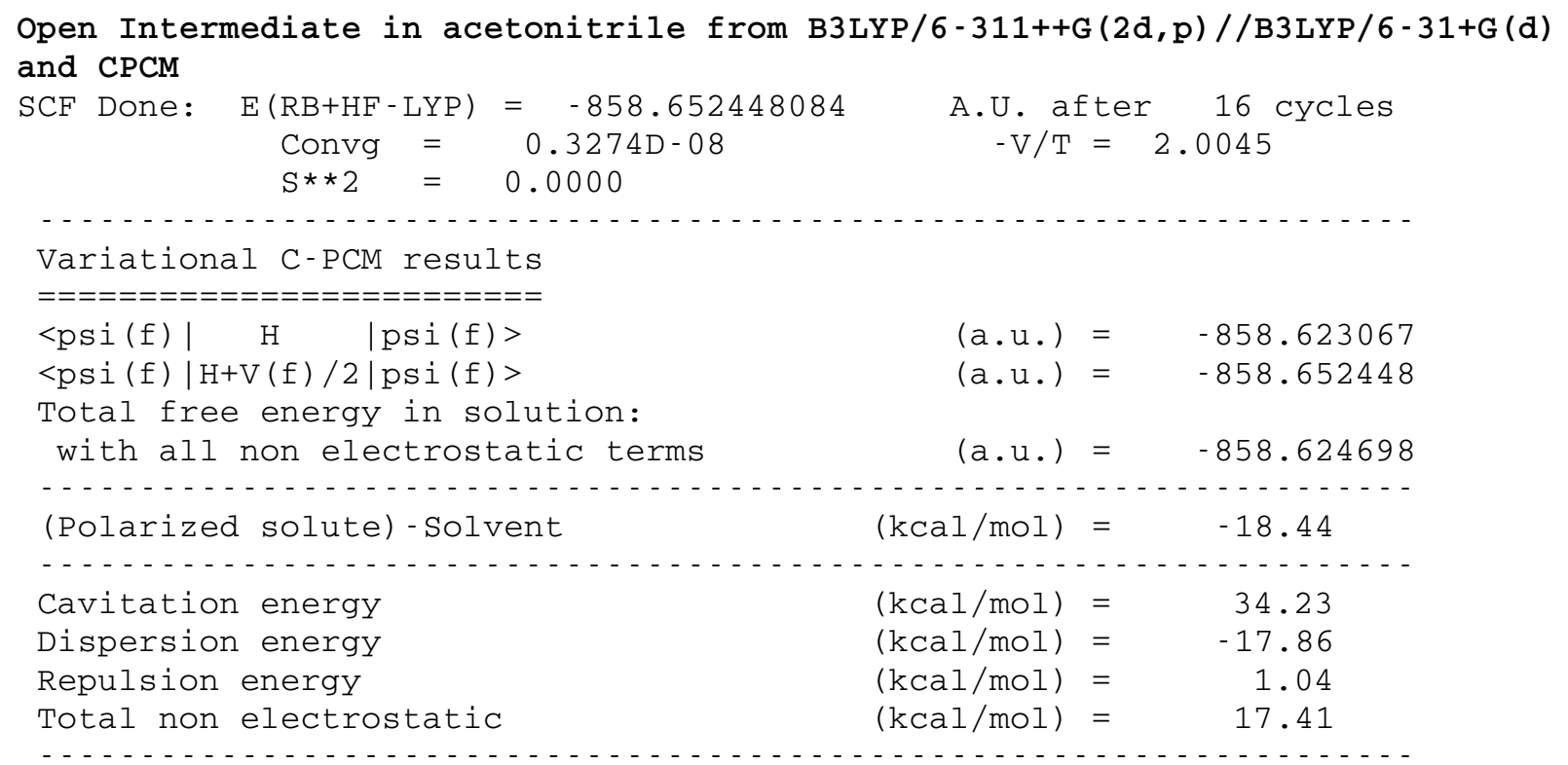

Intermediate isomerization TS in water from B3LYP/6-311++G $(2 d, p) / / B 3 L Y P / 6-$ $31+\mathrm{G}(\mathrm{d})$ and $\mathrm{CPCM}$

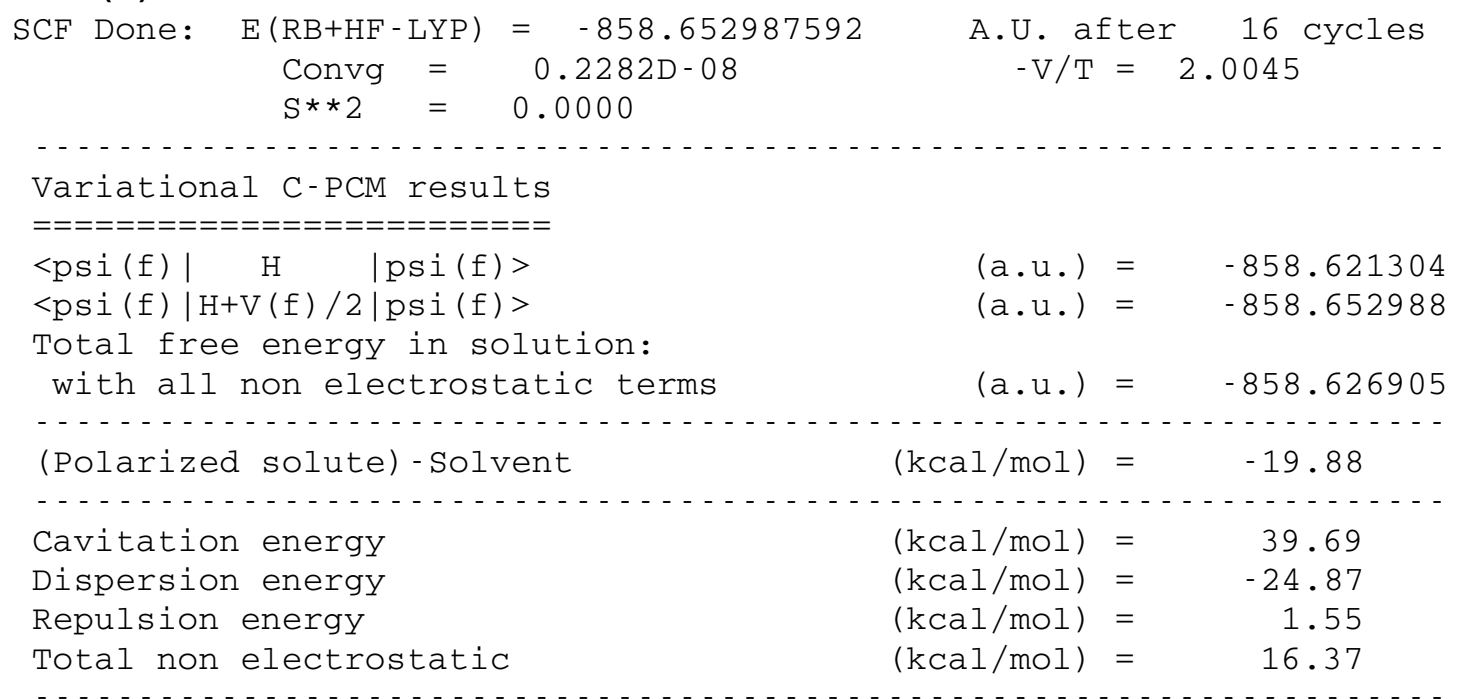

Intermediate isomerization TS in methanol from B3LYP/6-311++G(2d,p)//B3LYP/6$31+\mathrm{G}(\mathrm{d})$ and $\mathrm{CPCM}$ 


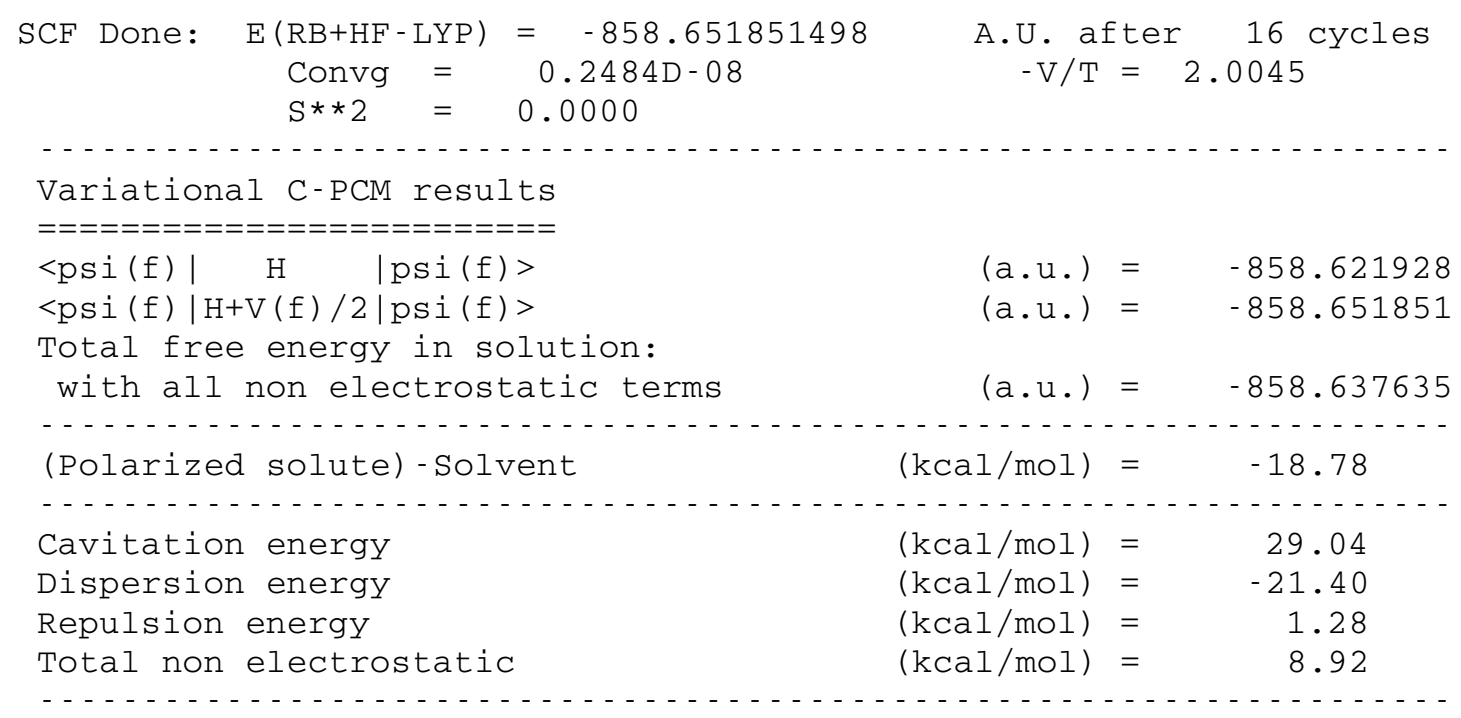

Intermediate isomerization TS in DMSO from B3LYP/6-311++G $(2 \mathrm{~d}, \mathrm{p}) / / \mathrm{B} 3 \mathrm{LYP} / 6-$ $31+\mathrm{G}(\mathrm{d})$ and $\mathrm{CPCM}$

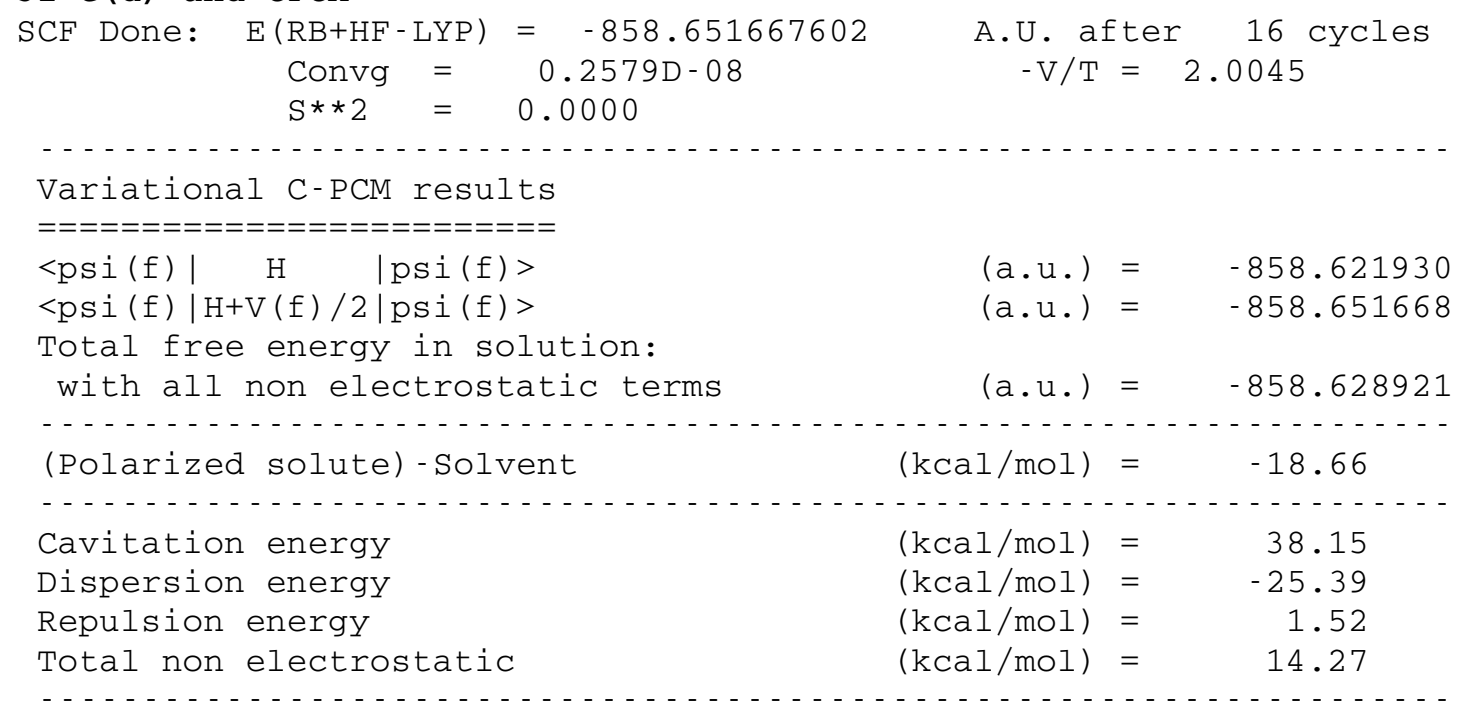

Intermediate isomerization TS in acetonitrile from B3LYP/6$311++G(2 d, p) / / B 3 L Y P / 6-31+G(d)$ and $C P C M$

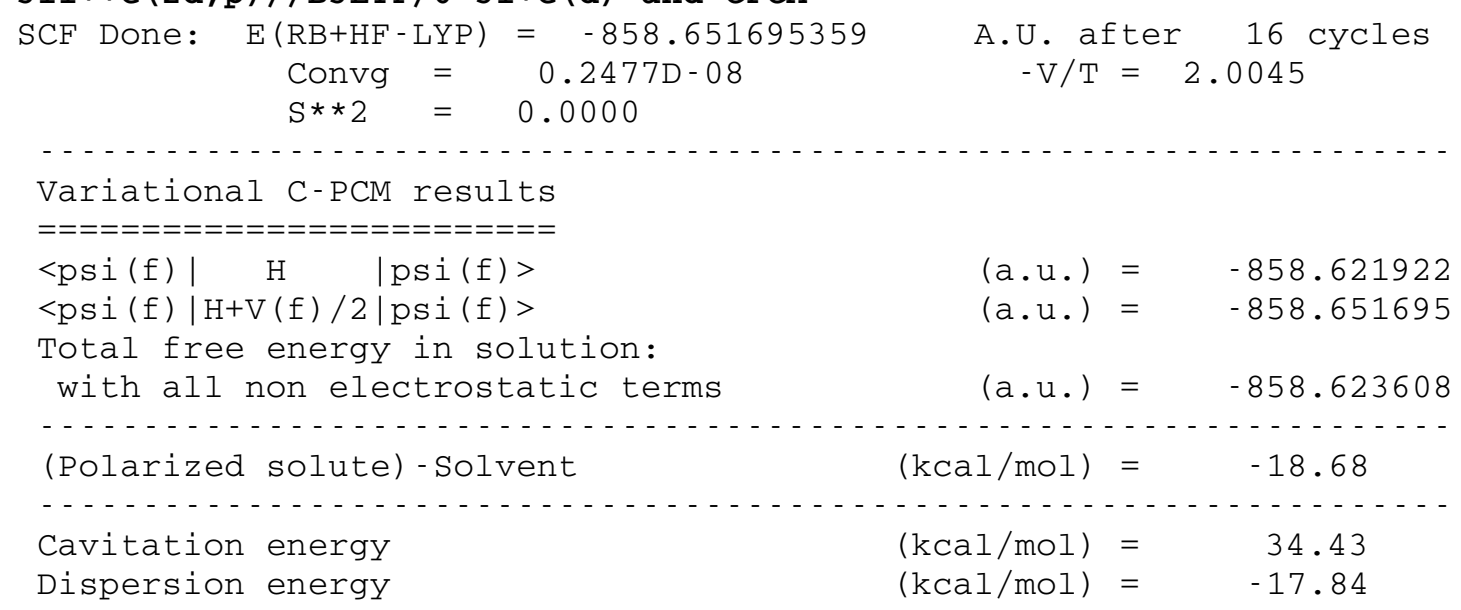




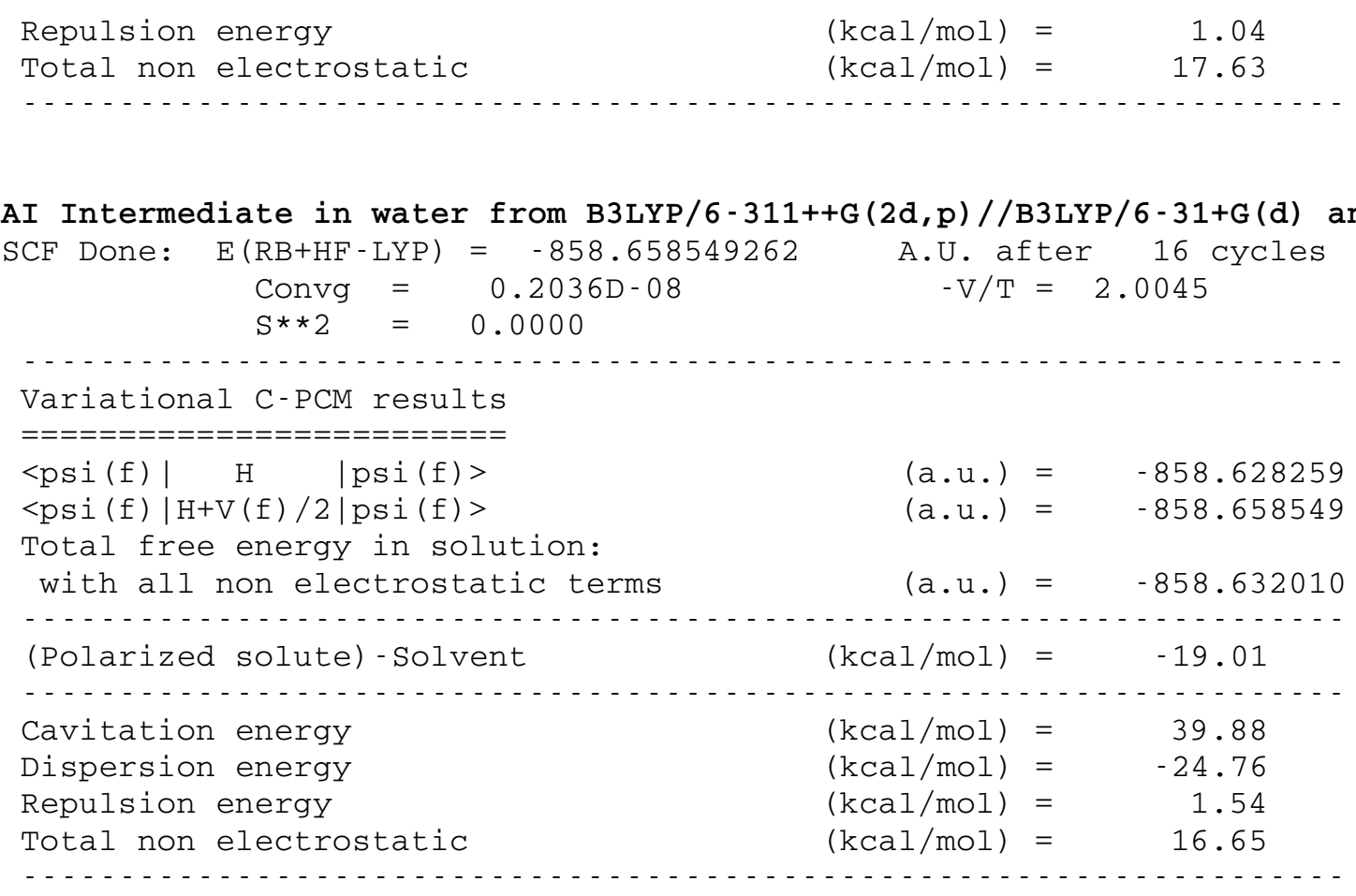

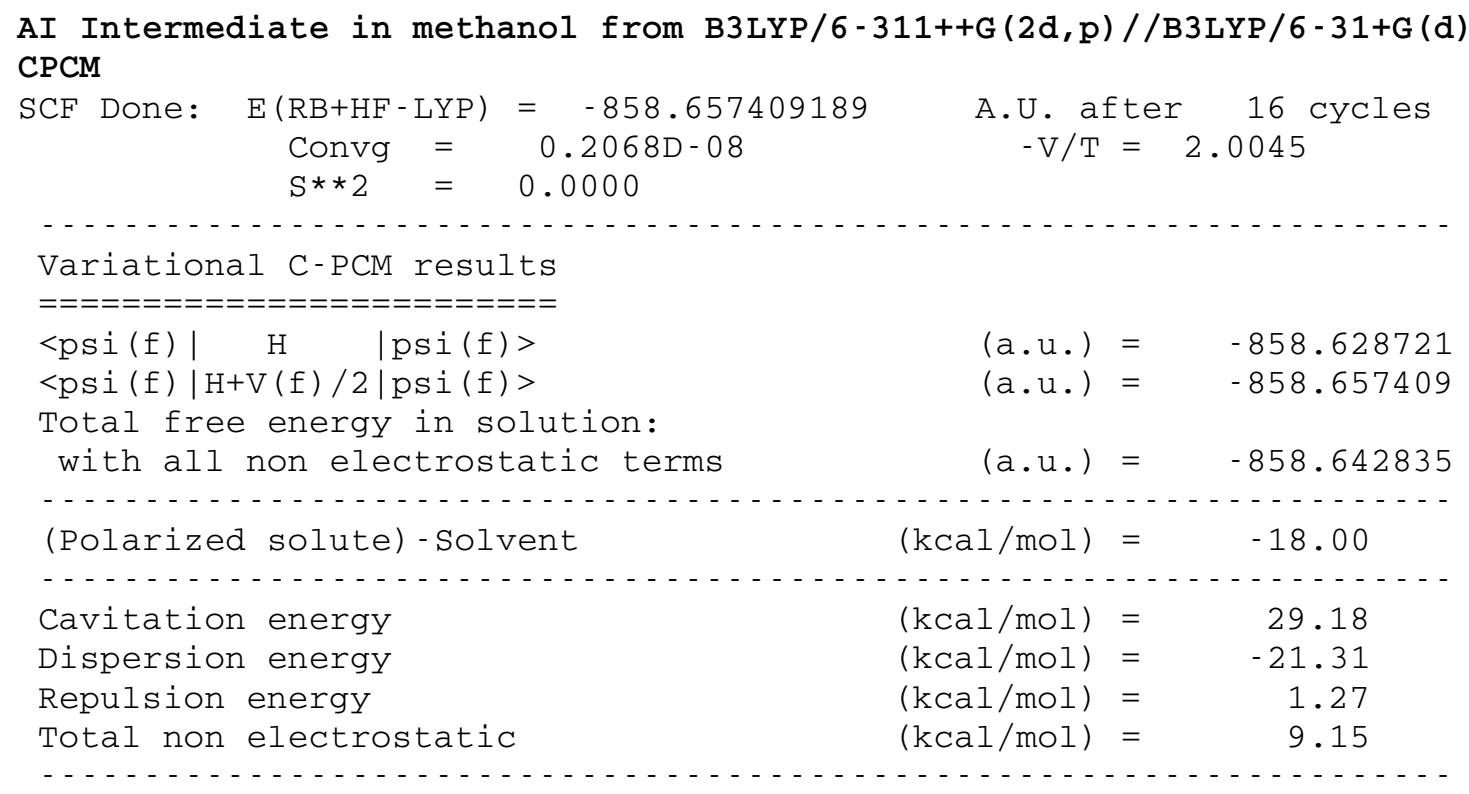

AI Intermediate in dmso from B3LYP/6-311++G $(2 d, p) / / B 3 L Y P / 6-31+G(d)$ and CPCM
$\mathrm{SCF}$ Done: $\mathrm{E}(\mathrm{RB}+\mathrm{HF}-\mathrm{LYP})=-858.657032412$ A.U. after 16 cycles

Convg $=0.2174 \mathrm{D}-08 \quad-\mathrm{V} / \mathrm{T}=2.0045$
$\mathrm{S} * * 2=0.0000$

Variational C-PCM results

$==================$

<psi(f)| H $\mid \operatorname{psi}(f)>\quad$ (a.u.) $=\quad-858.628716$

<psi(f) $|\mathrm{H}+\mathrm{V}(\mathrm{f}) / 2| \mathrm{psi}(\mathrm{f})>\quad$ (a.u.) $=\quad-858.657032$

Total free energy in solution:

with all non electrostatic terms (a.u.) = -858.633842

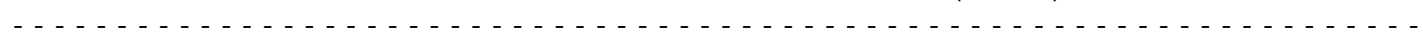




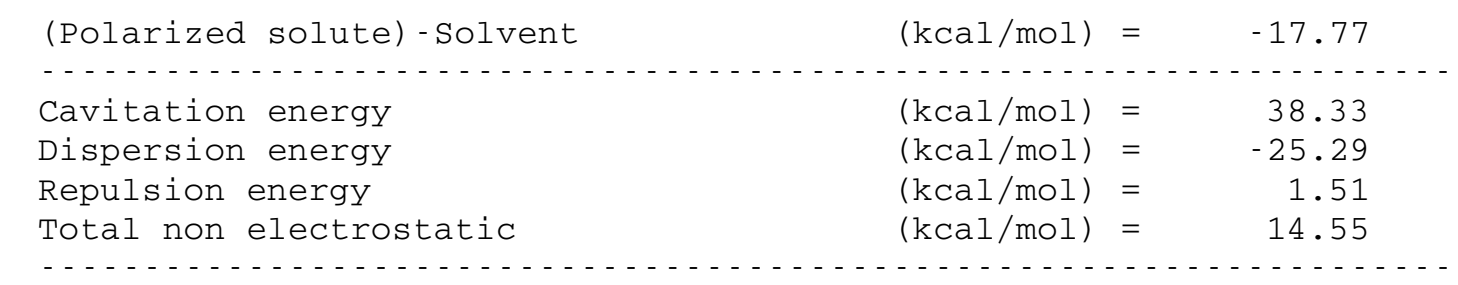

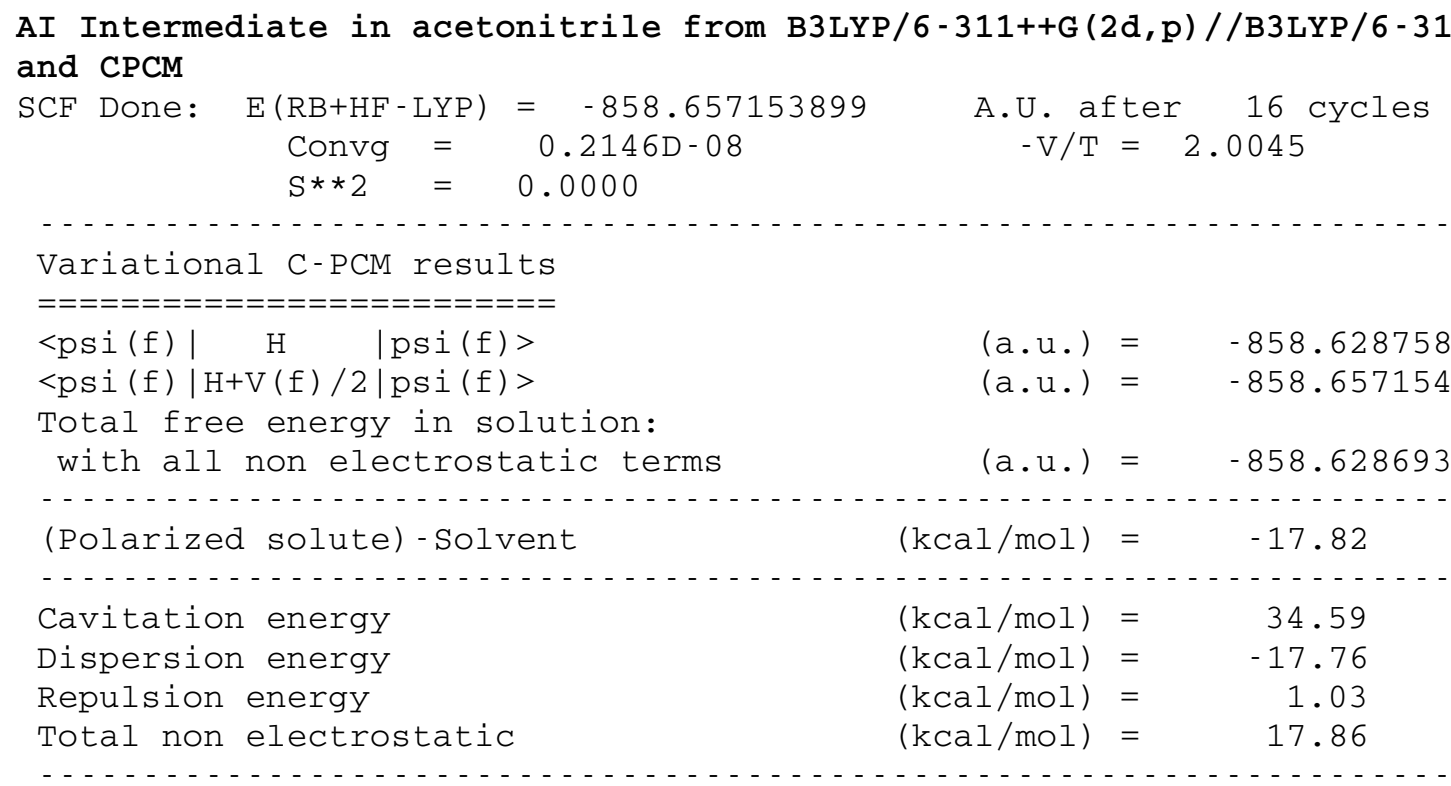

TS $a$ abs. in water from B3LYP/6-311++G $(2 \mathrm{~d}, \mathrm{p}) / / \mathrm{B} 3 \mathrm{LYP} / 6-31+\mathrm{G}(\mathrm{d})$ and CPCM $\mathrm{SCF}$ Done: $\mathrm{E}(\mathrm{RB}+\mathrm{HF}-\mathrm{LYP})=-858.645355118$ A.U. after 16 cycles Convg $=0.3277 \mathrm{D}-08 \quad-\mathrm{V} / \mathrm{T}=2.0045$ $\mathrm{S} * * 2=0.0000$

Variational C-PCM results $==================$

$<\operatorname{psi}(\mathrm{f})|\mathrm{H} \quad| \operatorname{psi}(\mathrm{f})>\quad\left(\mathrm{a} . \mathrm{u}_{\text {. }}\right)=-858.619590$ $<\operatorname{psi}(\mathrm{f})|\mathrm{H}+\mathrm{V}(\mathrm{f}) / 2| \operatorname{psi}(\mathrm{f})>\quad(\mathrm{a} . \mathrm{u})=$. Total free energy in solution:

with all non electrostatic terms (a.u.) $=-858.619781$

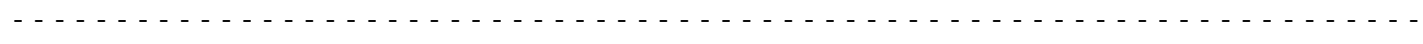

$($ Polarized solute) - Solvent $\quad(\mathrm{kcal} / \mathrm{mol})=-16.17$

Cavitation energy $\quad(\mathrm{kcal} / \mathrm{mol})=39.34$

Dispersion energy $\quad(\mathrm{kcal} / \mathrm{mol})=-24.84$

Repulsion energy $\quad(\mathrm{kcal} / \mathrm{mol})=1.55$

Total non electrostatic $\quad(\mathrm{kcal} / \mathrm{mol})=16.05$

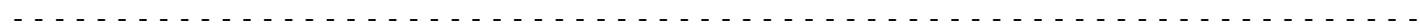

$\mathrm{TS}_{\mathrm{abs}}$. in methanol from B3LYP/6-311++G $(2 \mathrm{~d}, \mathrm{p}) / / \mathrm{B} 3 \mathrm{LYP} / 6-31+\mathrm{G}(\mathrm{d})$ and CPCM $\mathrm{SCF}$ Done: $\mathrm{E}(\mathrm{RB}+\mathrm{HF}-\mathrm{LYP})=-858.644500704$ A.U. after 15 cycles Convg $=0.6254 \mathrm{D}-08 \quad-\mathrm{V} / \mathrm{T}=2.0045$ $\mathrm{S} * * 2=0.0000$

Variational C-PCM results

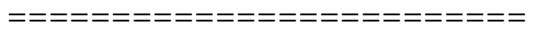

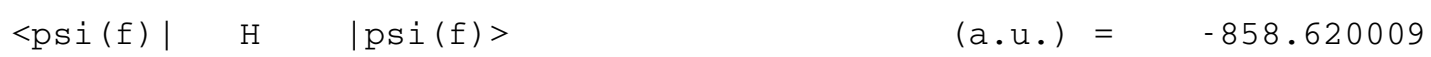




\begin{tabular}{|c|c|c|c|}
\hline$<\operatorname{psi}(f)|H+V(f) / 2| \operatorname{psi}(f)>$ & $(\mathrm{a} \cdot \mathrm{u} \cdot)$ & $=$ & -858.644501 \\
\hline \multicolumn{4}{|l|}{ Total free energy in solution: } \\
\hline with all non electrostatic terms & $(\mathrm{a} \cdot \mathrm{u})$. & $=$ & -858.630672 \\
\hline 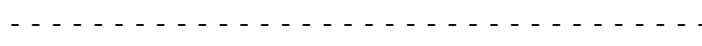 & $\ldots \ldots$ & - & $\ldots \ldots$ \\
\hline (Polarized solute)-Solvent & $(\mathrm{kcal} / \mathrm{mol})$ & $=$ & -15.37 \\
\hline 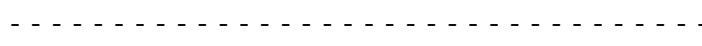 & $\ldots \ldots$ & - & $\ldots \ldots$ \\
\hline Cavitation energy & $(\mathrm{kcal} / \mathrm{mol})$ & $=$ & 28.78 \\
\hline Dispersion energy & $(\mathrm{kcal} / \mathrm{mol})$ & $=$ & $-21 \cdot 38$ \\
\hline Repulsion energy & $(\mathrm{kcal} / \mathrm{mol})$ & $=$ & 1.28 \\
\hline Total non electrostatic & $(\mathrm{kcal} / \mathrm{mol})$ & $=$ & 8.68 \\
\hline
\end{tabular}

$\mathrm{TS}_{\mathrm{abs}}$. in dmso from B3LYP/6-311++G $(2 \mathrm{~d}, \mathrm{p}) / / \mathrm{B} 3 \mathrm{LYP} / 6-31+\mathrm{G}(\mathrm{d})$ and CPCM $\mathrm{SCF}$ Done: $\mathrm{E}(\mathrm{RB}+\mathrm{HF}-\mathrm{LYP})=-858.644451663$ A.U. after 15 cycles
Convg $=0.5901 \mathrm{D}-08$
$-\mathrm{V} / \mathrm{T}=2.0045$

$\mathrm{S} * * 2=0.0000$

Variational C-PCM results

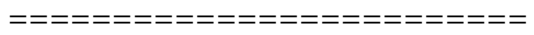

<psi(f)| $\mathrm{H} \quad \mid \operatorname{psi}(\mathrm{f})>\quad$ (a.u.) $=-858.619957$

$<\operatorname{psi}(f)|H+V(f) / 2| \operatorname{psi}(f)>\quad$ (a.u.) $=\quad-858.644452$

Total free energy in solution:

with all non electrostatic terms (a.u.) = -858.622259

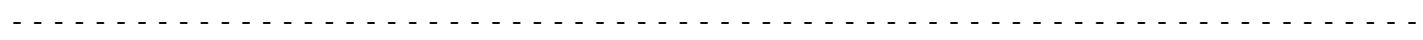

(Polarized solute)-Solvent $\quad(\mathrm{kcal} / \mathrm{mol})=-15.37$

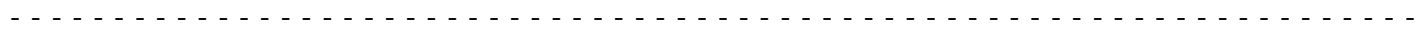

Cavitation energy

Dispersion energy

Repulsion energy

Total non electrostatic

$(\mathrm{kcal} / \mathrm{mol})=37.80$

$(\mathrm{kcal} / \mathrm{mol})=-25.39$

$(\mathrm{kcal} / \mathrm{mol})=1.52$

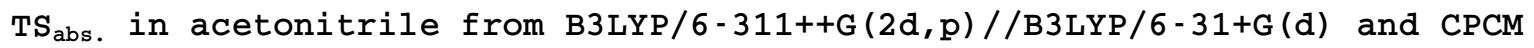

$\mathrm{SCF}$ Done: $\mathrm{E}(\mathrm{RB}+\mathrm{HF}-\mathrm{LYP})=-858.644377611$ A.U. after 15 cycles

Convg $=0.6097 \mathrm{D}-08 \quad-\mathrm{V} / \mathrm{T}=2.0045$

$\mathrm{S} * * 2=0.0000$

Variational C-PCM results

$===================$

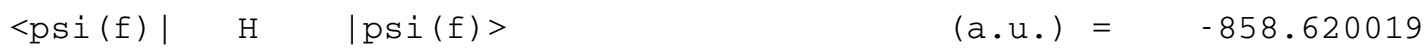

$<\operatorname{psi}(f)|H+V(f) / 2| p s i(f)>$

Total free energy in solution:

with all non electrostatic terms (a.u.) = -858.616786

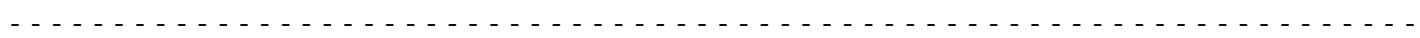

(Polarized solute)-Solvent $\quad(\mathrm{kcal} / \mathrm{mol})=-15.28$

- . . . . . . . . . . . . . . . . . . . . . . . . . . . . . . . . .

Cavitation energy $\quad(\mathrm{kcal} / \mathrm{mol})=34.11$

Dispersion energy $\quad(\mathrm{kcal} / \mathrm{mol})=-17.84$

Repulsion energy $\quad(\mathrm{kcal} / \mathrm{mol})=1.04$

Total non electrostatic $\quad(\mathrm{kcal} / \mathrm{mol})=17.31$

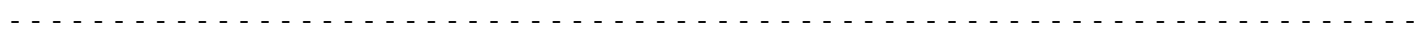

Ene Product in water from B3LYP/6-311++G (2d,p)//B3LYP/6-31+G(d) and CPCM

$\mathrm{SCF}$ Done: $\mathrm{E}(\mathrm{RB}+\mathrm{HF}-\mathrm{LYP})=-622.723279168$

A.U. after 13 cycles

$\begin{array}{llll}\text { Convg } & =0.5871 \mathrm{D}-08 & -\mathrm{V} / \mathrm{T}=2.0042 \\ \mathrm{~S} * * 2 & =0.0000 & \end{array}$

- 


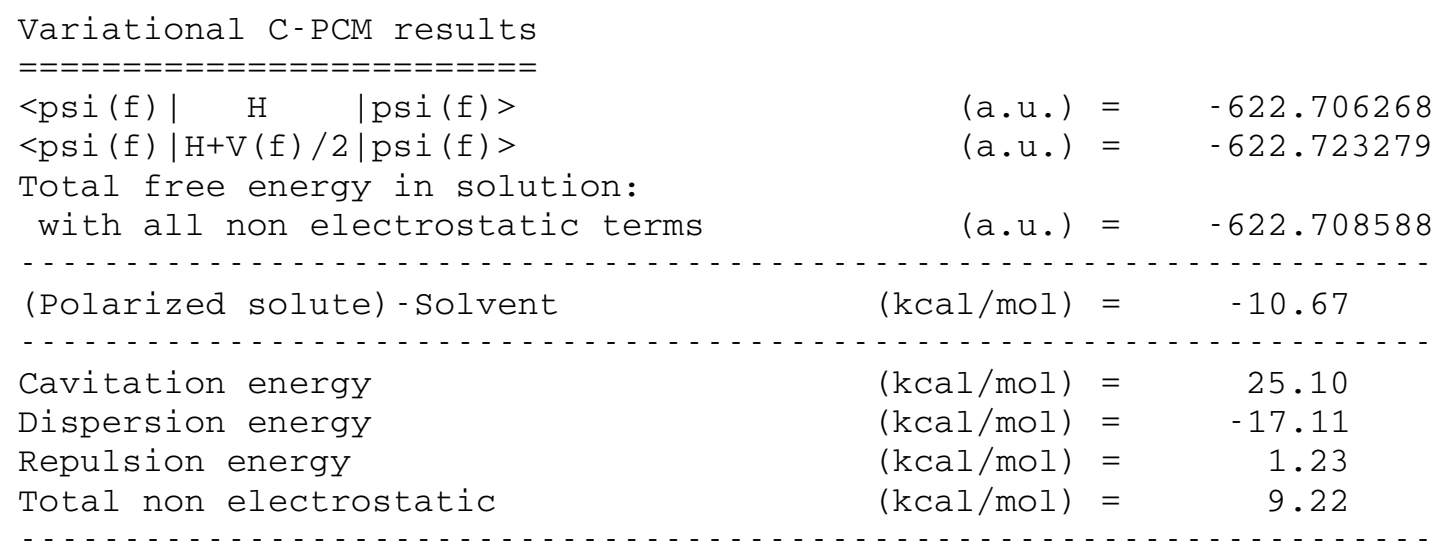

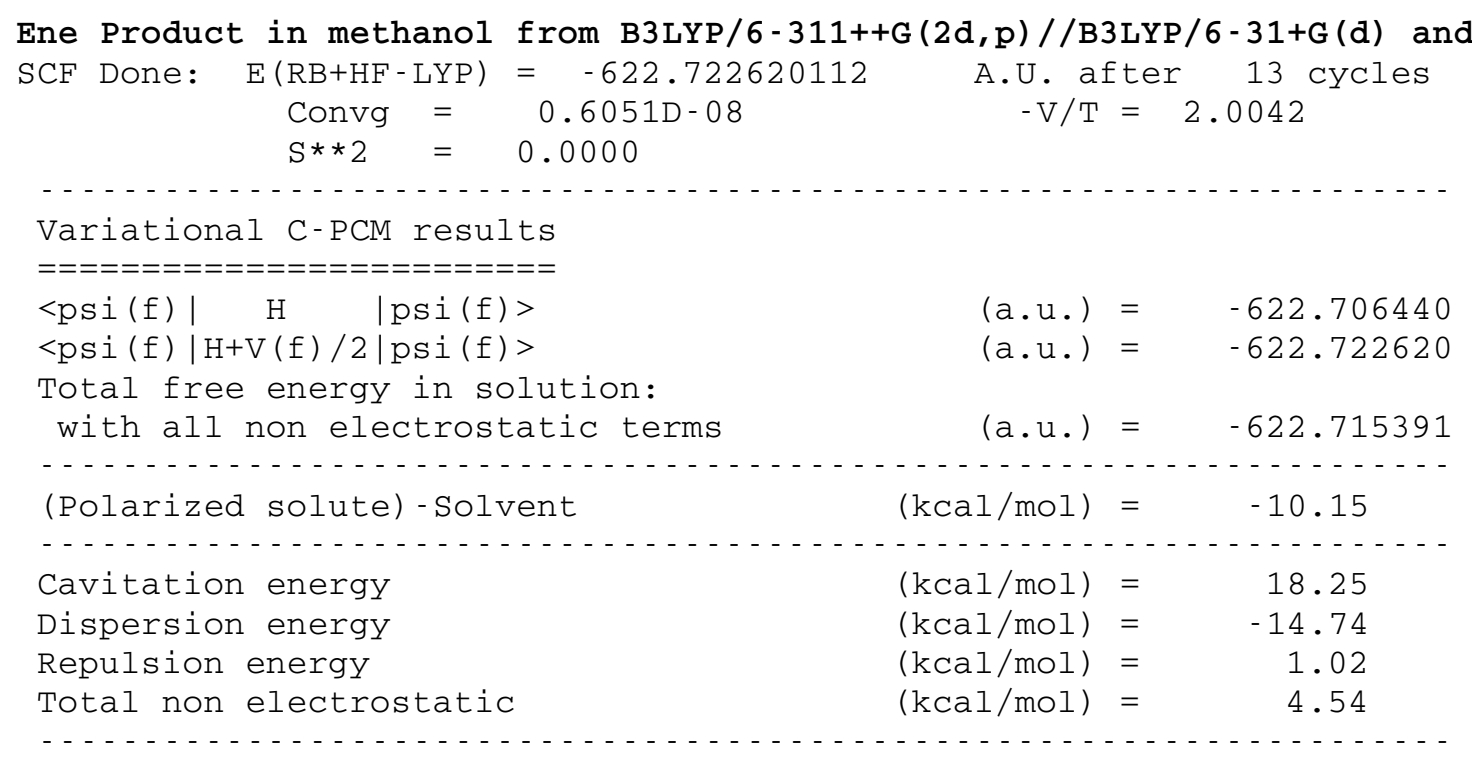

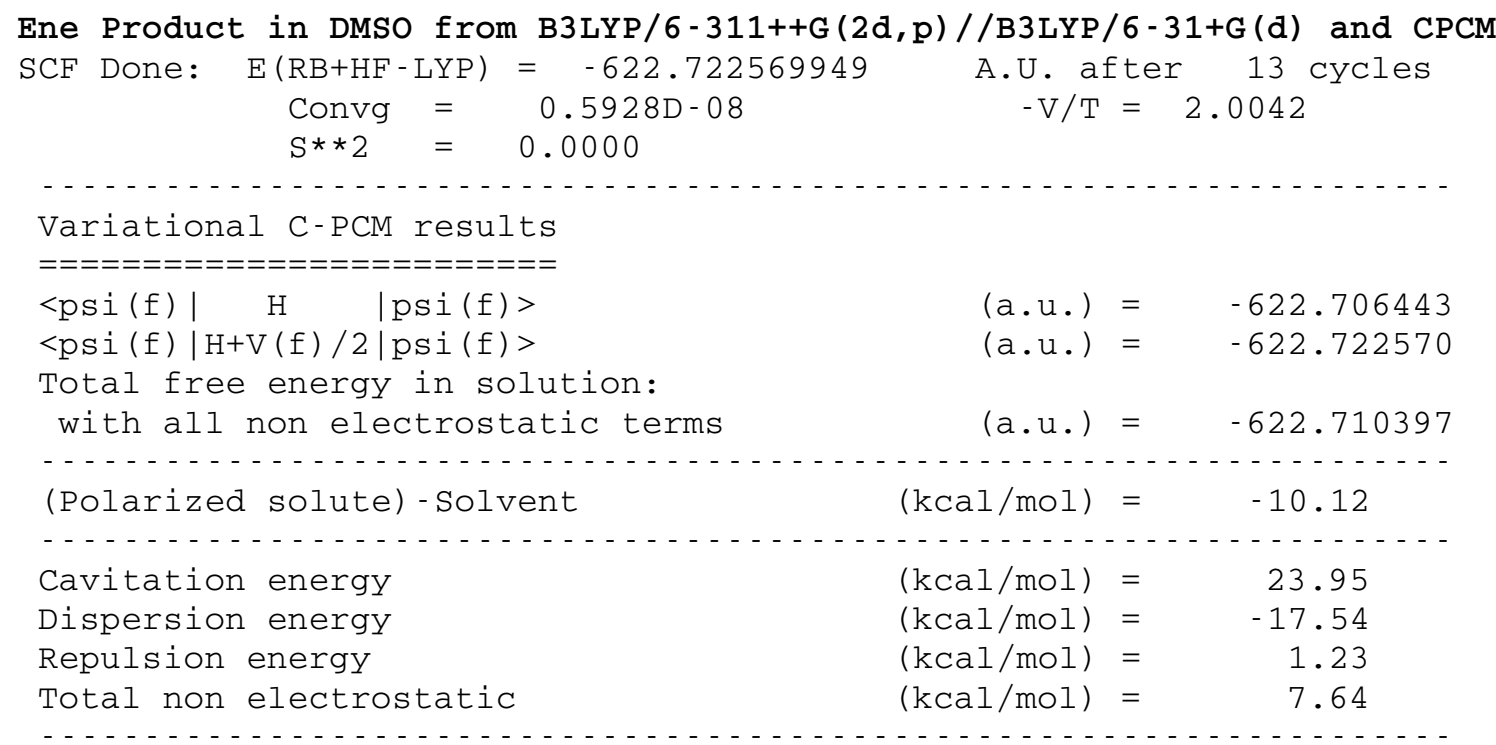

Ene Product in acetonitrile from B3LYP/6-311++G $(2 d, p) / / B 3 L Y P / 6-31+G(d)$ and CPCM 


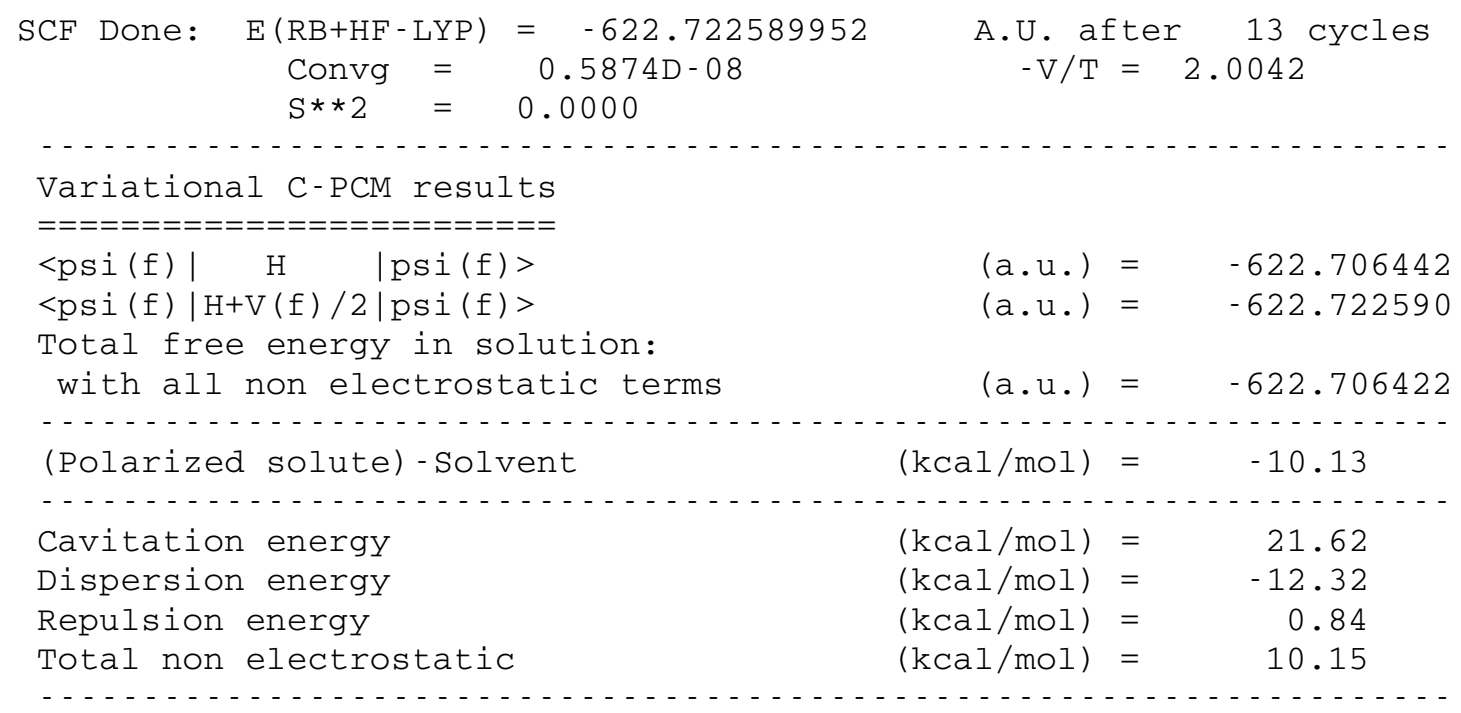

\section{Open Intermediate from UB3LYP/6-31+G(d)}

$1 \backslash 1 \backslash$ ASN_ALTIX - ALTIX7 \FOpt \UB3LYP \6-31+G (d) \C14H17N3O2\AUBOXA \30 - May - 2 $007 \backslash 1 \backslash \backslash \#$ UB3LYP/6-31+G(d) opt=(noeigen, z-matrix) guess=mix $\backslash \backslash \mathrm{PD} \operatorname{PhTAD} \backslash \backslash$ $0,1 \backslash N \backslash N, 1, B 1 \backslash \mathrm{C}, 2, \mathrm{~B} 2,1, \mathrm{~A} 1 \backslash \mathrm{C}, 1, \mathrm{~B} 3,2, \mathrm{~A} 2,3, \mathrm{D} 1,0 \backslash \mathrm{N}, 4, \mathrm{~B} 4,1, \mathrm{~A} 3,2, \mathrm{D} 2,0 \backslash 0,3, \mathrm{~B} 5$, $2, \mathrm{~A} 4,1, \mathrm{D} 3,0 \backslash 0,4, \mathrm{~B} 6,1, \mathrm{~A} 5,2, \mathrm{D} 4,0 \backslash \mathrm{C}, 5, \mathrm{~B} 7,4, \mathrm{~A} 6,1, \mathrm{D} 5,0 \backslash \mathrm{C}, 8, \mathrm{~B} 8,5, \mathrm{~A} 7,4, \mathrm{D} 6,0 \backslash \mathrm{C}$ $, 8, \mathrm{~B} 9,5, \mathrm{~A} 8,4, \mathrm{D} 7,0 \backslash \mathrm{C}, 9, \mathrm{~B} 10,8, \mathrm{~A} 9,5, \mathrm{D} 8,0 \backslash \mathrm{C}, 10, \mathrm{~B} 11,8, \mathrm{~A} 10,5, \mathrm{D} 9,0 \backslash \mathrm{C}, 12, \mathrm{~B} 12,1$ $0, \mathrm{~A} 11,8, \mathrm{D} 10,0 \backslash \mathrm{H}, 10, \mathrm{~B} 13,8, \mathrm{~A} 12,5, \mathrm{D} 11,0 \backslash \mathrm{H}, 9, \mathrm{~B} 14,8, \mathrm{~A} 13,5, \mathrm{D} 12,0 \backslash \mathrm{H}, 12, \mathrm{~B} 15,10$ , $\mathrm{A} 14,8, \mathrm{D} 13,0 \backslash \mathrm{H}, 11, \mathrm{~B} 16,9, \mathrm{~A} 15,8, \mathrm{D} 14,0 \backslash \mathrm{H}, 13, \mathrm{~B} 17,12, \mathrm{~A} 16,10, \mathrm{D} 15,0 \backslash \mathrm{C}, 1, \mathrm{~B} 18,4$

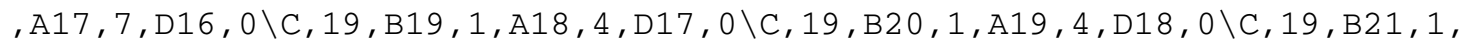
$\mathrm{A} 20,4, \mathrm{D} 19,0 \backslash \mathrm{C}, 20, \mathrm{~B} 22,19, \mathrm{~A} 21,1, \mathrm{D} 20,0 \backslash \mathrm{C}, 20, \mathrm{~B} 23,19, \mathrm{~A} 22,1, \mathrm{D} 21,0 \backslash \mathrm{H}, 21, \mathrm{~B} 24,1$ 9 , A23 , 1 , D22 , $\backslash \backslash \mathrm{H}, 21, \mathrm{~B} 25,19, \mathrm{~A} 24,1, \mathrm{D} 23,0 \backslash \mathrm{H}, 21, \mathrm{~B} 26,19, \mathrm{~A} 25,1, \mathrm{D} 24,0 \backslash \mathrm{H}, 22, \mathrm{~B} 27$

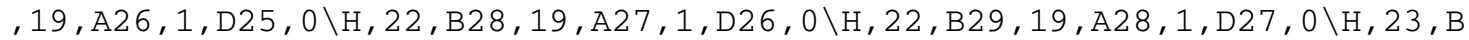
$30,20, \mathrm{~A} 29,19, \mathrm{D} 28,0 \backslash \mathrm{H}, 23, \mathrm{~B} 31,20, \mathrm{~A} 30,19, \mathrm{D} 29,0 \backslash \mathrm{H}, 23, \mathrm{~B} 32,20, \mathrm{~A} 31,19, \mathrm{D} 30,0 \backslash \mathrm{H}$ , $24, \mathrm{~B} 33,20, \mathrm{~A} 32,19, \mathrm{D} 31,0 \backslash \mathrm{H}, 24, \mathrm{~B} 34,20, \mathrm{~A} 33,19, \mathrm{D} 32,0 \backslash \mathrm{H}, 24, \mathrm{~B} 35,20, \mathrm{~A} 34,19, \mathrm{D} 3$ $3,0 \backslash \backslash \mathrm{B} 1=1.34636488 \backslash \mathrm{B} 2=1.38009099 \backslash \mathrm{B} 3=1.43229675 \backslash \mathrm{B} 4=1.37732588 \backslash \mathrm{B} 5=1.2196$ $3407 \backslash B 6=1.22219002 \backslash B 7=1.42797436 \backslash B 8=1.40000167 \backslash B 9=1.39974926 \backslash B 10=1.395$ $92747 \backslash \mathrm{B} 11=1.39604439 \backslash \mathrm{B} 12=1.39739956 \backslash \mathrm{B} 13=1.08344781 \backslash \mathrm{B} 14=1.08389224 \backslash \mathrm{B} 15=$ $1.08678748 \backslash \mathrm{B} 16=1.08684434 \backslash \mathrm{B} 17=1.08680649 \backslash \mathrm{B} 18=1.50948976 \backslash \mathrm{B} 19=1.50766557$ $\backslash \mathrm{B} 20=1.53569573 \backslash \mathrm{B} 21=1.53391578 \backslash \mathrm{B} 22=1.48482967 \backslash \mathrm{B} 23=1.49012797 \backslash \mathrm{B} 24=1.089$ $3803 \backslash \mathrm{B} 25=1.09556894 \backslash \mathrm{B} 26=1.09427253 \backslash \mathrm{B} 27=1.09480799 \backslash \mathrm{B} 28=1.08899342 \backslash \mathrm{B} 29=1$ $.09587994 \backslash \mathrm{B} 30=1.10063786 \backslash \mathrm{B} 31=1.10989756 \backslash \mathrm{B} 32=1.0933206 \backslash \mathrm{B} 33=1.09346317 \backslash \mathrm{B}$ $34=1.0981833 \backslash B 35=1.10521601 \backslash A 1=106.61988085 \backslash A 2=112.56352802 \backslash A 3=103.700$ $60823 \backslash A 4=126.96240052 \backslash A 5=126.88046245 \backslash A 6=125.167478 \backslash A 7=120.01246362 \backslash A 8$ $=119.54945662 \backslash \mathrm{A} 9=119.51340731 \backslash \mathrm{A} 10=119.44698879 \backslash \mathrm{A} 11=120.53927942 \backslash \mathrm{A} 12=11$ $9.92485641 \backslash \mathrm{A} 13=120.05838803 \backslash \mathrm{A} 14=119.27658258 \backslash \mathrm{A} 15=119.31361262 \backslash \mathrm{A} 16=120$. $211496 \backslash A 17=124.12362868 \backslash A 19=109.03073336 \backslash A 20=110.57608063 \backslash A 21=117.9370$ $917 \backslash A 22=121.09229905 \backslash A 23=112.92833805 \backslash A 24=108.26543896 \backslash A 25=109.9699358$ $5 \backslash A 26=109.34273864 \backslash A 27=112.98048613 \backslash A 28=108.85944123 \backslash A 29=111.85611128 \backslash$ $\mathrm{A} 30=110.30765702 \backslash \mathrm{A} 31=112.11716173 \backslash \mathrm{A} 32=111.27720049 \backslash \mathrm{A} 33=111.78504935 \backslash \mathrm{A} 3$ $4=110.89352322 \backslash \mathrm{D} 1=-2.40169449 \backslash \mathrm{D} 2=2.7863965 \backslash \mathrm{D} 3=-177.5237265 \backslash \mathrm{D} 4=-178.592$ $20093 \backslash D 5=-183.51626828 \backslash D 6=-39.19610222 \backslash D 7=141.27048141 \backslash D 8=180.07365262$ $\backslash \mathrm{D} 9=-180.62181148 \backslash \mathrm{D} 10=0.51899808 \backslash \mathrm{D} 11=0.74403355 \backslash \mathrm{D} 12=0.84014201 \backslash \mathrm{D} 13=180$ $.45685355 \backslash \mathrm{D} 14=-179.75550548 \backslash \mathrm{D} 15=-180.19761961 \backslash \mathrm{D} 16=-20.49376516 \backslash \mathrm{D} 17=-65$ $.52266241 \backslash \mathrm{D} 18=175.3469423 \backslash \mathrm{D} 19=55.45398814 \backslash \mathrm{D} 20=-72.32583997 \backslash \mathrm{D} 21=99.5849$ $2465 \backslash \mathrm{D} 22=55.64342965 \backslash \mathrm{D} 23=176.75645843 \backslash \mathrm{D} 24=-64.80416579 \backslash \mathrm{D} 25=62.97469719$ $\backslash \mathrm{D} 26=-57.68558483 \backslash \mathrm{D} 27=-179.19959478 \backslash \mathrm{D} 28=-71.0274468 \backslash \mathrm{D} 29=44.74233133 \backslash \mathrm{D} 3$ 


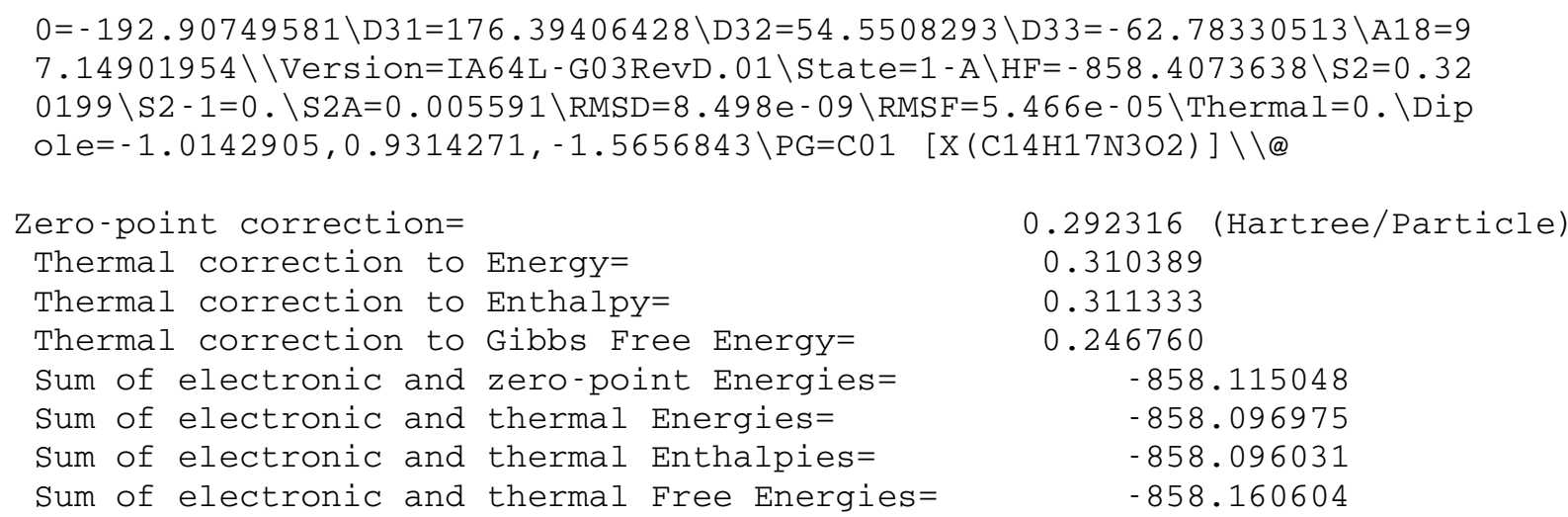

\section{Gaussian 03 Reference}

Gaussian 03, Revision D.01,

M. J. Frisch, G. W. Trucks, H. B. Schlegel, G. E. Scuseria,

M. A. Robb, J. R. Cheeseman, J. A. Montgomery, Jr., T. Vreven,

K. N. Kudin, J. C. Burant, J. M. Millam, S. S. Iyengar, J. Tomasi,

V. Barone, B. Mennucci, M. Cossi, G. Scalmani, N. Rega,

G. A. Petersson, H. Nakatsuji, M. Hada, M. Ehara, K. Toyota,

R. Fukuda, J. Hasegawa, M. Ishida, T. Nakajima, Y. Honda, O. Kitao,

H. Nakai, M. Klene, X. Li, J. E. Knox, H. P. Hratchian, J. B. Cross,

V. Bakken, C. Adamo, J. Jaramillo, R. Gomperts, R. E. Stratmann,

O. Yazyev, A. J. Austin, R. Cammi, C. Pomelli, J. W. Ochterski,

P. Y. Ayala, K. Morokuma, G. A. Voth, P. Salvador, J. J. Dannenberg,

V. G. Zakrzewski, S. Dapprich, A. D. Daniels, M. C. Strain,

O. Farkas, D. K. Malick, A. D. Rabuck, K. Raghavachari,

J. B. Foresman, J. V. Ortiz, Q. Cui, A. G. Baboul, S. Clifford,

J. Cioslowski, B. B. Stefanov, G. Liu, A. Liashenko, P. Piskorz,

I. Komaromi, R. L. Martin, D. J. Fox, T. Keith, M. A. Al-Laham,

C. Y. Peng, A. Nanayakkara, M. Challacombe, P. M. W. Gill,

B. Johnson, W. Chen, M. W. Wong, C. Gonzalez, and J. A. Pople, Gaussian, Inc., Wallingford CT, 2004 . 